\title{
Vortex multipoles in two-layer rotating shallow-water flows
}

\author{
By JEAN-MICHEL BAEY ${ }^{1}$ AND XAVIER CARTON \\ ${ }^{1}$ ATLANTIDE, Brest, France \\ ${ }^{2}$ Laboratoire de Physique des Océans, IFREMER, Brest, France
}

(Received 20 December 2000 and in revised form 15 November 2001)

The stability of elliptically perturbed circular vortices is investigated in a two-layer shallow-water model, with constant background rotation. The fluid is bounded above and below by rigid and flat surfaces. The linear stability analysis shows that elliptical perturbations are most unstable for moderate Burger numbers and vorticity shears. Shorter waves dominate for more sheared vortices. Shallow-water and quasigeostrophic growth rates exhibit a striking similarity, except at each end of the Burger number domain. There, cyclones (anticyclones) with finite Rossby numbers are more (less) unstable than their quasi-geostrophic counterparts. A simple model gives a first-order trend for this bias.

Nonlinear model runs with initially perturbed vortices also show the similarity between the two dynamics. In these runs, elliptically deformed vortices stabilize as stationary rotating tripoles for moderate linear instability; on the other hand, strongly unstable vortices break as dipoles. During these nonlinear processes, energy transfers indicate that barotropic instability is at least as active as the baroclinic one. For tripole formation, the modal analysis of the perturbation exhibits a dominant contribution of the original wave and of the mean flow correction. The ageostrophic and divergent parts of the flow are respectively weak and negligible. The Lighthill equation proves that few internal gravity waves are generated during tripole formation or dipolar breaking. Finally, the effects of triangular perturbations on circular vortices and the formation of quadrupoles are briefly addressed.

\section{Previous work and problem setting}

Vortices are long-lived features of rotating flows, both natural and experimental This robustness is due in part to their resistance to external perturbations. Such perturbations often consist of large-scale shear or strain fields, which induce antisymmetric or elliptical deformations on circular vortices. The initial value problem of an elliptically perturbed vortex, in a two-layer shallow-water flow, is considered here. This problem has already been the subject of many studies, for quasi-geostrophic and shallow-water flows.

In two-dimensional incompressible flows, both numerical and laboratory experiments showed that elliptically deformed, shielded vortices $\dagger$ can transform into longlived tripoles if the perturbation amplitude is moderate and if the mean horizontal vorticity shear is not too large (Carton, Flierl \& Polvani 1989; van Heijst, Kloosterziel \& Williams 1991; Orlandi \& van Heijst 1992). Once formed, these tripoles

$\dagger$ Shielded vortices possess a sign reversal in the radial vorticity profile; they are isolated if their total circulation vanishes. 
are stationary and uniformly rotating for many turnover periods (Carton \& Legras 1994). In the long term, they can break asymmetrically into a monopole and a dipole; this evolution is favoured by viscosity and by the $\beta$-effect (Carton \& Legras 1994). More complex, less robust, multipoles can be obtained by perturbing strongly sheared circular vortices with higher wavenumbers (Carnevale \& Kloosterziel 1994; Morel \& Carton 1994).

In two-layer quasi-geostrophic flows, stationary tripoles with various vertical structures (the more robust being barotropic) have been obtained by perturbing circular vortices elliptically (Corréard \& Carton 1999). Steady baroclinic tripoles can also result from the head-on collision of finite-area hetons (Sokolovskiy \& Verron 2000). On the other hand, vertically antisymmetric vortices form periodically oscillating, counter-rotating ellipses (Carton \& McWilliams 1996).

The nonlinear evolution of elliptically perturbed vortices has also been addressed in rotating shallow-water flows. In one-and-a-half layer reduced-gravity flows, ageostrophic effects favour anticyclone rather than cyclone stability at finite Rossby number, while small Burger numbers stabilize all vortices (Stegner \& Dritschel 2000). Ford (1994) found a mixed type of instability with Rossby waves interacting with an internal gravity wave; this instability was either weak or dominated by inertial instability. In two-layer flows, warm eddies, with Gaussian radial profile, are usually more stable than their cold counterparts (Dewar \& Killworth 1995). These large warm eddies are all the more stable as their shallow and deep flows are co-rotating. With cubic exponential profiles, warm co-rotating vortices are essentially stable, while cold vortices are not stabilized by a deep like-signed flow (Killworth, Blundell \& Dewar 1997; Dewar, Blundell \& Killworth 1999).

The present work is concerned with the formation and evolution of tripoles in a two-layer rotating shallow-water flow. Several questions are addressed:

What is the influence of the horizontal and vertical vorticity shears, of the Rossby and Burger numbers, and of vortex polarity on the linear and nonlinear instability of elliptically perturbed, circular vortices on the $f$-plane?

When tripoles are formed, what is their three-dimensional structure? How important are ageostrophic or divergent flows? Are these tripoles stationary in a rotating frame of reference? Are they long-lived and resistant to perturbations?

This paper is organized as follows: $\S 2$ presents the model equations, initial conditions and numerical implementation. In $\S 3$, the linear stability of circular vortices is described and the influence of physical parameters investigated. Parity bias is given special attention. Then the finite-amplitude regimes observed in initial-value simulations are classified and tripole formation is analysed $(\S 4)$ : modal decomposition, energy budgets, and final stationary states are presented. Ageostrophic and divergent flows are investigated. Finally $(\S 5)$, the formation of more complex multipoles is briefly described and conclusions are given.

\section{The model}

\subsection{Model equations}

The dimensionless two-layer shallow-water equations, with uniform background rotation, rigid lid and flat bottom, are written in polar coordinates:

$$
\begin{aligned}
R o \frac{\mathrm{d} \boldsymbol{u}_{j}}{\mathrm{~d} t}+\left(1+R o \frac{v_{j}}{r}\right) \boldsymbol{k} & \wedge \boldsymbol{u}_{j}=-\nabla p_{j}, \\
\frac{\mathrm{d} h_{j}}{\mathrm{~d} t}+h_{j} \nabla \cdot \boldsymbol{u}_{j}=0 &
\end{aligned}
$$


where $\boldsymbol{u}_{j}=\left(u_{j}, v_{j}\right), h_{j}$ and $p_{j}$ are the (radial, azimuthal) velocity, thickness and pressure normalized by density, in the $j$ th layer (counted from the top). The layer thickness is

$$
h_{j}=H_{j}\left(1+(-1)^{j} \frac{R o}{B u} \frac{\eta}{\hat{H}_{j}}\right),
$$

where $R o=U / f L$ and $B u=g^{\prime} H / f^{2} L^{2}$ are the Rossby and Burger numbers, $f$ is the dimensional background rotation and $\hat{H}_{j}=H_{j} / H$ are the normalized layer thicknesses at rest. The elevation of the density interface is $\eta$.

The Lagrangian derivative is

$$
\frac{\mathrm{d}}{\mathrm{d} t}=\partial_{t}+u_{j} \partial_{r}+\frac{v_{j}}{r} \partial_{\theta}
$$

These equations can be combined to prove the conservation of potential vorticity anomaly (computed from a state of rest):

$$
\frac{\mathrm{d} q_{j}}{\mathrm{~d} t}=0, \quad q_{j}=\frac{\zeta_{j}+(-1)^{j+1} \eta /\left(B u \hat{H}_{j}\right)}{1+(-1)^{j}(R o / B u)\left(\eta / \hat{H}_{j}\right)} .
$$

2.2. Nonlinear model implementation and initial conditions

The numerical implementation of these equations is performed via a pseudo-spectral projection/truncation in a biperiodic domain. The number of nodes in each direction is 128 for the general parameter search and 256 for specific case analysis. The momentum equations are given a minimum biharmonic viscosity compatible with numerical stability (namely $v_{4}=2 \times 10^{-7}$ at $128^{2}$ resolution; physically, the dissipative timescale is 600 turnover periods at the core of a vortex with $R o=0.1$ ). An Asselin filter helps stabilize fast waves. Total mass conservation is ensured by subtracting the barotropic divergent velocity from the flow field at each time step. The barotropic streamfunction is also used to compute the surface pressure diagnostically (Baraille \& Filatoff 1995). Finally, though most results presented here correspond to equal layer thickness at rest, many simulations have been performed with $\hat{H}_{1}=0.2, \hat{H}_{2}=0.8$, a case for which triadic interactions of the first baroclinic mode are non-zero (Flierl 1978); they yield qualitatively similar results.

The mean flow is a circular vortex, with a power exponential radial profile and annular shielding in the relative vorticity:

$$
\bar{\zeta}_{1}=\left(1-\frac{1}{2} \alpha r^{\alpha}\right) \exp \left(-r^{\alpha}\right), \quad \bar{\zeta}_{2}=\kappa \bar{\zeta}_{1} .
$$

This vortex is isolated and its velocity and pressure are in cyclogeostrophic balance:

$$
R o \frac{\bar{v}_{j}^{2}}{r}+\bar{v}_{j}=\frac{\mathrm{d} \bar{p}_{j}}{\mathrm{~d} r} .
$$

A general investigation of this vortex instability has been performed by varying $\alpha \in[2,9], l \in[1,4], R o \in[0.05,1], B u \in[0,5], \kappa \in[-1,1]$. Only specific aspects of this whole parametric investigation are given hereafter.

\section{Linear stability}

\subsection{Barotropic and baroclinic instabilities}

The linear instability of a normal-mode disturbance over the circular vortex is computed:

$$
\left(u_{j}^{\prime}, v_{j}^{\prime}, p_{j}^{\prime}, h_{j}^{\prime}\right)=\left(u_{0 j}, v_{0 j}, p_{0 j}, h_{0 j}\right)(r) \exp [\mathrm{i} l(\theta-c t)]
$$


the elliptical perturbation corresponds to $l=2$, the triangular one to $l=3$. The momentum and continuity equations (1) and (2) are linearized to yield

$$
\begin{aligned}
& \bar{h}_{j}\left(c-\frac{\bar{v}_{j}}{r}\right) \frac{\mathrm{d}^{2} p_{0 j}}{\mathrm{~d} r^{2}}+\left(c-\frac{\bar{v}_{j}}{r}\right)\left(\left(\frac{1}{r}-\frac{V_{j 2}}{V_{j 1}}\right) \bar{h}_{j}+\frac{\mathrm{d} \bar{h}_{j}}{\mathrm{~d} r}\right) \frac{\mathrm{d} p_{0 j}}{\mathrm{~d} r} \\
& \quad+\left(\frac{\bar{h}_{j}}{r}\left(\frac{1}{R o}\left(\frac{1}{r}+\frac{V_{j 2}}{V_{j 1}}\right)-\frac{l^{2} c}{r}-\frac{2}{r} \frac{\mathrm{d} \bar{v}_{j}}{\mathrm{~d} r}+\left(\frac{4+l^{2}}{r}+2 \frac{V_{j 2}}{V_{j 1}}\right) \frac{\bar{v}_{j}}{r}\right)-\left(\frac{\bar{h}_{j}}{r}+\frac{\mathrm{d} \bar{h}_{j}}{\mathrm{~d} r}\right)\right. \\
& \left.\quad \times\left(\frac{1}{R o r}+2 \frac{\bar{v}_{j}}{r^{2}}\right)\right) p_{0 j}+(-1)^{j} V_{j 1}\left(c-\frac{\bar{v}_{j}}{r}\right) \frac{R o^{2}}{B u}\left(p_{01}-p_{02}\right)=0
\end{aligned}
$$

with

$$
\begin{gathered}
V_{j 1}=\left(\frac{1}{R o}+2 \frac{\bar{v}_{j}}{r}\right)\left(\frac{1}{R o}+\frac{\bar{v}_{j}}{r}+\frac{d \bar{v}_{j}}{d r}\right)-l^{2}\left(c-\frac{\bar{v}_{j}}{r}\right)^{2} \\
V_{j 2}=\left(\frac{1}{R o}+2 \frac{\bar{v}_{j}}{r}\right) \frac{\mathrm{d}^{2} \bar{v}_{j}}{\mathrm{~d} r^{2}}+\frac{1}{r}\left(\frac{\mathrm{d} \bar{v}_{j}}{\mathrm{~d} r}-\frac{\bar{v}_{j}}{r}\right)+\left(2 \frac{\mathrm{d} \bar{v}_{j}}{\mathrm{~d} r}+\left(4-2 l^{2}\right) \frac{\bar{v}_{j}}{r}+\frac{3}{R o}+2 l^{2} c\right) .
\end{gathered}
$$

The boundary condition at infinity is no normal flow:

$$
\left(c-\frac{\bar{v}_{j}}{r}\right) \frac{\mathrm{d} p_{0 j}}{\mathrm{~d} r}-\left(\frac{1}{R o r}+2 \frac{\bar{v}_{j}}{r^{2}}\right) p_{0 j}=0 .
$$

At $r=0$, regularity of the two linear differential equations leads to simpler equations:

$$
\frac{\mathrm{d} p_{0 j}}{\mathrm{~d} r}-\frac{l^{2}}{r} p_{0 j}=0 .
$$

This problem is discretized in $r$ and the growth rates of the perturbation $\sigma=l \operatorname{Im}(c)$ are computed by a Newton-Raphson method. Setting $p_{01}(r=0)=1$, this method uses first guesses for $c$ and $c_{1}=p_{02}(r=0)$ which are either the quasi-geostrophic values (for small $R o$, large $B u$ ) or the result of the previous calculation when available (with the closest value of $R o, B u$ ). This method advects $p_{0 j}$ and $\mathrm{d} p_{0 j} / \mathrm{d} r$ from $r=0$ to large $r$, where the boundary condition of no normal flow is usually not satisfied at first iteration. By computing the variation of this discrepancy with respect to $c$ and $c_{1}$, a correction is introduced for these two values to satisfy the boundary conditions after a few iterations. This method fails to converge for cyclones at small $B u$, even when such small increments in $B u$ as $10^{-6}$ are used from one calculation to the following one. This can be attributed to the very fast variation of $c$ with $B u$ in this case or to a change in the family of eigenmodes which alters $c_{1}$. For all other cases, this algorithm has been checked against results available in the literature with great accuracy.

\subsection{Inertial instability}

Inertial instability does not affect our mean flow for the range of parameters given at the end of $\S 2$. Indeed, following Holton (1992), an inertial instability criterion for circular flows can be derived. The radial displacement of a potentially unstable fluid particle obeys the equation

$$
\frac{\mathrm{d}^{2} \delta r}{\mathrm{~d} r^{2}}=-\left(1+R o \Omega_{j}\right)\left(1+R o \zeta_{j}\right) \delta r,
$$

with $\Omega_{j}=\bar{v}_{j} / r$. The flow will be unstable if $\left(1+R o \Omega_{j}\right)\left(1+R o q_{j}\right)<0$. This implies that $R o<-1$ and/or $|\kappa|>1$ which is not the case here. 

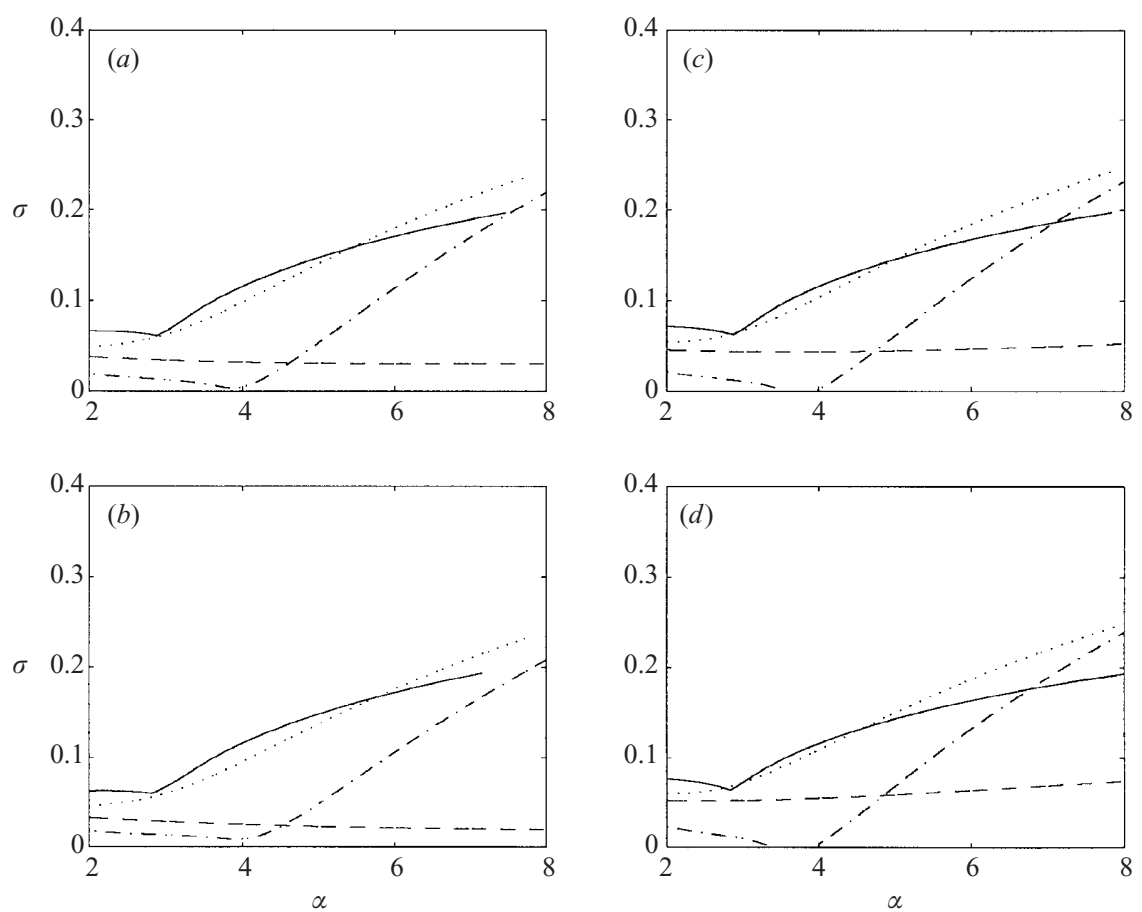

FIGURE 1. Influence of the Rossby number on the linear instability of circular vortices: growth rates versus $\alpha$, for $B u=0.25, \kappa=0, \hat{H}_{1}=0.5$. The left-hand column $(a, b)$ is for anticyclones, the right-hand one for cyclones $(c, d)$. The upper row $(a, c)$ is for shallow-water vortices with $R o= \pm 0.1$, the lower row $(b, d)$ with $R o= \pm 0.25$. The modes are $l=1$ (dashed line), $l=2$ (solid line), $l=3$ (dotted line), $l=4$ (dot-dash line).

\subsection{Variation of growth rates, most unstable wavelengths and the nature of the instability with physical parameters}

The elliptical perturbation $(l=2)$ is the most unstable for moderate horizontal vorticity shears $(\alpha \in[2,5])$ and for order-unity values of the Burger number (see figure 1). This predominance does not depend on the Rossby number nor on vortex polarity. For increasingly stronger barotropic instability $(\kappa \sim 1, \alpha \gg 1, B u \geqslant 1)$, or baroclinic instability $(\kappa \leqslant 0, \alpha \sim 2, B u \ll 1)$, shorter and shorter waves become more unstable (particularly so as the mean flow is baroclinic). In most cases, the growth rate of the asymmetric perturbation $(l=1)$ is half the size of that for the dominant mode and it is significant only in regimes of strong baroclinic instability. These results are similar in the quasi-geostrophic framework and for unequal layer thicknesses (not shown).

The analysis of linear energy transfers between the mean flow and the perturbation was performed for many cases (see Appendix A for the expressions for energies and energy fluxes). A typical case is that of surface-intensified, cubic exponential vortices with an elliptical perturbation, for two values of the Rossby number $(R o=0.1,0.5$; figure not shown). For small Burger numbers, the perturbation kinetic energy comes from the potential energy of the mean flow and from the baroclinic conversion (between potential and kinetic energy of the perturbation). On increasing the Burger number, the kinetic energy of the perturbation is fed by that of the mean flow and 

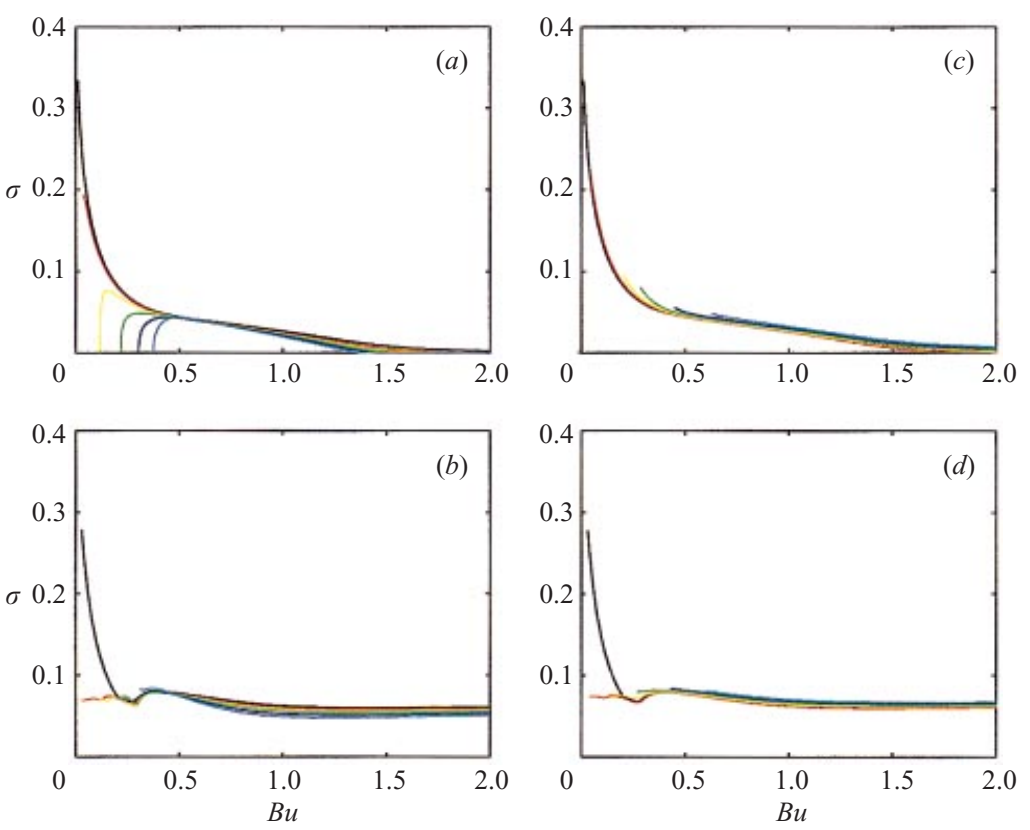

FIGURE 2. Influence of the horizontal shear on the linear instability of surface-intensified $(\kappa=0)$, circular vortices: growth rates of mode $l=2$ versus $B u$ for various values of $R o(R o=0.05$ : red; $R o=0.25$ : yellow; $R o=0.5$ : green; $R o=0.75$ : blue and $R o=1:$ pink). The steepness of the vorticity profile increases from top to bottom: $(a, c) \alpha=2,(b, d) \alpha=3 ;(a, b)$ anticyclones, $(c, d)$ cyclones; $\hat{H}_{1}=0.5$.
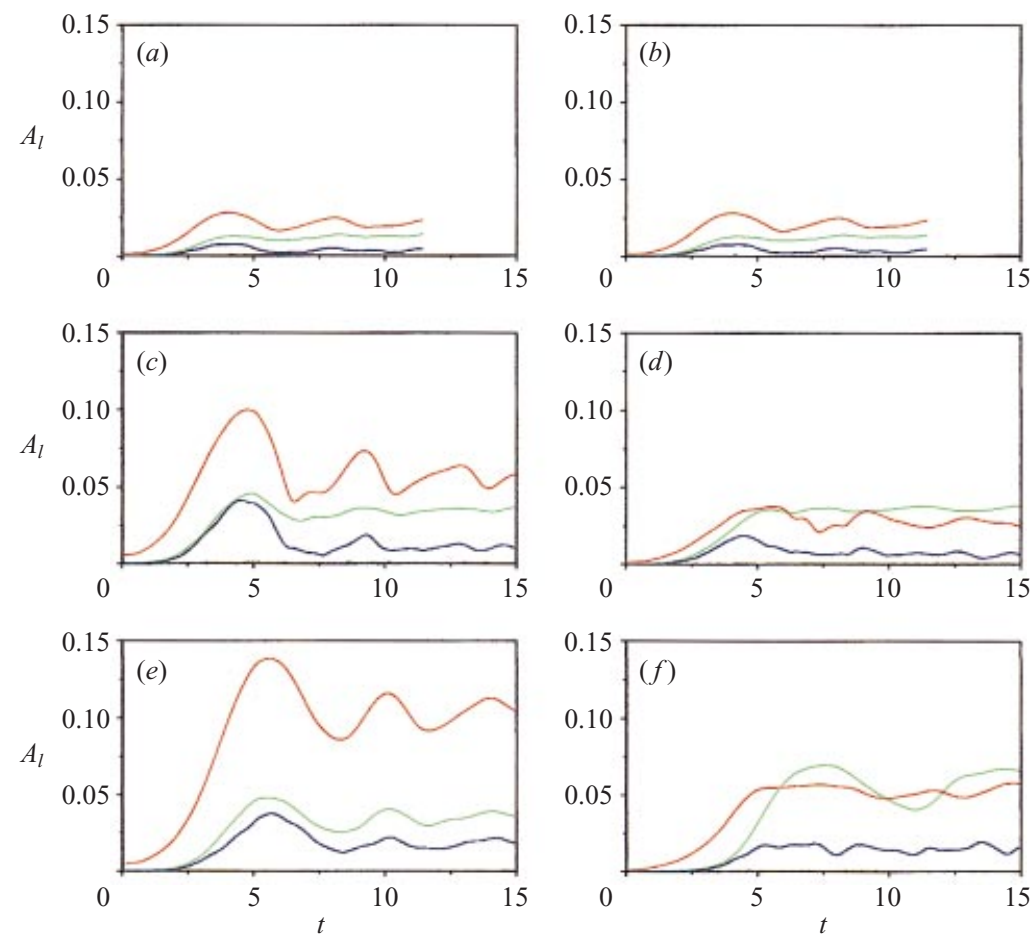

FIGURE 7. Evolution of the angular modes for the evolution of an anticyclonic vortex into: $(a, b)$ a barotropic tripole $(R o=0.1, B u=0.25, \kappa=1, \alpha=3, l=2) ;(c, d)$ a surface-intensified tripole $(R o=0.25, B u=0.25, \kappa=0.5, \alpha=3, l=2) ;(e, f)$ a baroclinic tripole $(R o=0.5, B u=1, \kappa=0$, $\alpha=3, l=2$ ). The various modes are $l=0$ (green line), $l=1$ (black line), $l=2$ (red line), $l=3$ (yellow line), $l=4$ (blue line). The upper (lower) layer is on the left-hand (right-hand) column. 


$\begin{array}{llllcllll}R o & -1.0 & -0.75 & -0.5 & -0.25 & 0.25 & 0.5 & 0.75 & 1.0 \\ B u_{F} & 0.375 & 0.305 & 0.219 & 0.116 & 0.2 & 0.285 & 0.45 & 0.63 \\ B u_{\text {outcrop }} & 0.34 & 0.278 & 0.2 & 0.108 & 0.123 & 0.26 & 0.412 & 0.58\end{array}$

TABLE 1. Comparison of Burger number values for zero growth rate (anticyclone) or lack of numerical convergence (cyclone) in the frontal limit, $B u_{F}$, with those for outcropping (interface deviation equal to layer thickness), $B u_{\text {outcrop }}$, for $\alpha=2, \kappa=0, l=2, \hat{H}_{1}=0.5$.

the baroclinic conversion is reversed. This confirms the predominance of barotropic or of baroclinic instability in the two extreme regimes as described above.

\subsection{Parity bias at small and large Burger numbers and onset of baroclinic instability}

We next analyse the difference in instability between cyclones and anticyclones, an effect absent from the quasi-geostrophic model results. To do so, we use a surfaceintensified vortex $(\kappa=0)$ with an elliptical perturbation; two horizontal shears $(\alpha=2,3)$ are considered, for which barotropic instability is weak or moderate in two-dimensional flows (Carton et al. 1989; Orlandi \& van Heijst 1992). On each plot of figure 2, growth rates obtained for different values of $R o$ are superimposed: a remarkable similarity between quasi-geostrophic and shallow-water model results is seen in most of the $B u$ domain. Nevertheless a noticeable asymmetry appears at small $B u$ (see frames $(a)$ and $(c)$ ) and a less intense one at large $B u$ (see frames $(b)$ and $(d)$ ). The first case corresponds to important frontal effects as soon as $R o / B u \sim 1$ (i.e. vertical deviations of the density interface are large for the mean vortex). Note that similar results are found for the triangular perturbation $(l=3)$, namely anticyclone stability for Gaussian vortices in the frontal limit, and cyclone/anticyclone asymmetry in the large $B u$ limit for strong horizontal shears $(\alpha=4)$.

\subsubsection{Small Burger number, frontal limit}

In this limit, we consider the values of $B u$ for which growth rates vanish (for anticyclones) or for which the Newton-Raphson method stops converging (for cyclones), called $B u_{F}$. Table 1 compares them with the values of $B u$ corresponding to the out/incropping of the mean vortex, $B u_{\text {outcrop }}$. We always have $B u_{F}>B u_{\text {outcrop }}$, but the two values are fairly close. This suggests that the asymmetry in the instability of cyclones and anticyclones in this regime could be connected with the strong deviation of density interfaces.

To investigate this connection, a simple model is derived (see Appendix B) from the linearized conservation of potential height anomaly (PHA, the inverse of potential vorticity). In the frontal limit, mean potential vorticity diverges and cannot be used for a linear stability analysis. We assume moreover that the perturbation is geostrophic; this assumption implies that $R o$ remains small (here we choose $R o \leqslant 0.4$ ); consequently $B u$ must be small enough to keep $R o / B u$ of order unity. Obviously, the simple model will only be a crude approximation of the shallow-water model.

Figure 3 shows the growth rates versus Burger number for cyclones and anticyclones in this simple model. Very weak asymmetry is observed at large $B u$, but cyclones become notably more unstable than anticyclones at small $B u$. This tendency is comparable with that of the shallow-water model. Two possible physical interpretations of this phenomenon are as follows:

(i) The decrease/increase of mean potential thickness below the vortex core is 


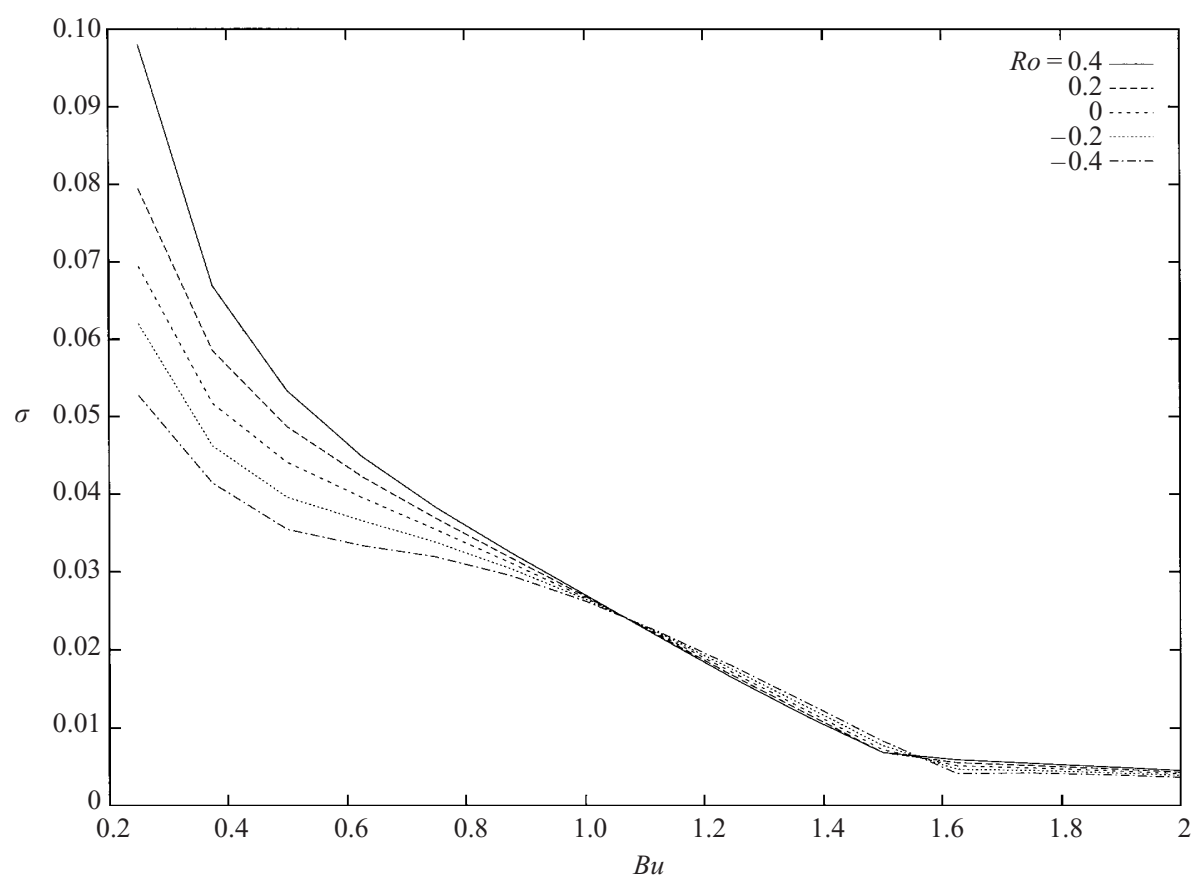

FiguRE 3. Growth rates of mode $l=2$ versus $B u$ in the simple model for the frontal limit. Plots for $R o=-0.4,-0.2,0,0.2,0.4 . \alpha=2, \kappa=0, \hat{H}_{1}=0.5$.

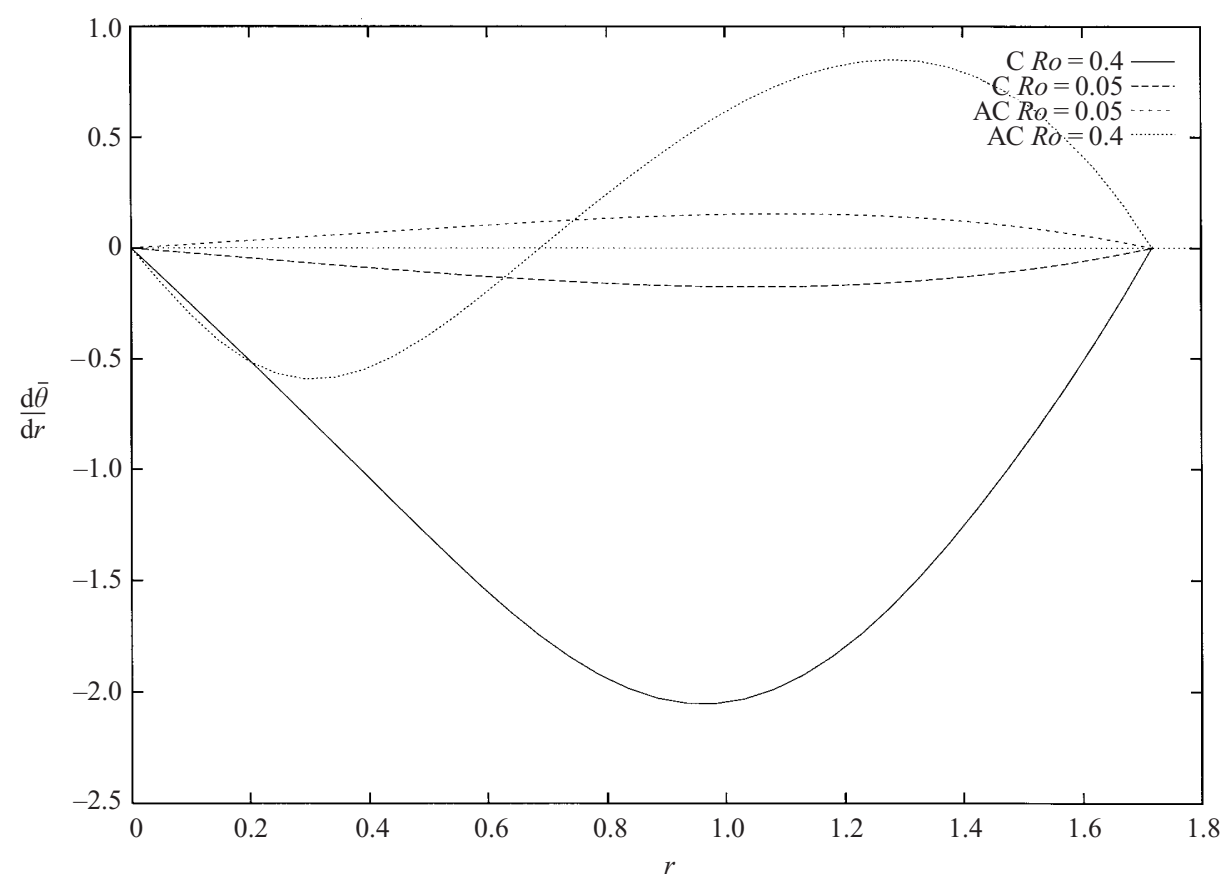

FIGURE 4. Radial gradient of mean potential height anomaly for $B u=0.25$ and $R o=0.4,0.05,-0.05,-0.4 . \alpha=2, \kappa=0, \hat{H}_{1}=0.5$. C denotes cyclone, AC anticyclone. 


$\begin{array}{lcccll}R o & -0.5 & -0.25 & -0.05 & 0.05 & 0.25 \\ B u_{c} & 1.56 & 1.69 & 1.84 & 2.19 & 2.46 \\ B u_{t h} & 1.6 & 1.7 & 1.9 & 2.1 & 2.8\end{array}$

TABLE 2. Comparison of critical Burger number for baroclinic instability found numerically $B u_{c}$, with that derived theoretically with a local deformation radius $B u_{t h}$ (same parameters as table 1).

associated with a weaker/stronger possibility of development of a perturbation in the lower layer, hence of a vertical phase shift necessary for baroclinic instability.

(ii) By analogy with quasi-geostrophic theory, the stronger the radial gradient of mean PHA is, the stronger the instability will be. Figure 4 shows this radial gradient for cyclones and anticyclones at $R o=0.05$ and $R o=0.4$. The PHA gradient for cyclones at small $R o$ is antisymmetrical with respect to that for anticyclones, hence the comparable growth rates for both vortex types. On the other hand, at larger Ro, the cyclone PHA gradient is large and negative, while the anticyclone gradient is smaller and changes sign. This is associated with the stronger instability of cyclones.

Our simple model does not reproduce the stabilization of anticyclones at small $B u$, which could be due to ageostrophic components of the velocity. Indeed, $(a)$ they grow with $R o$, and at first order correspond to a cyclostrophic correction (see Appendix $\mathrm{C}$ ) and $(b)$ they contribute to the decrease of anticyclone instability compared to that of cyclones, for a symmetric mean flow at large $B u$ (see below).

\subsubsection{Critical value of the Burger number for the onset of baroclinic instability}

The onset of baroclinic instability occurs at smaller values of $B u$ for anticyclones than for cyclones in the shallow-water model (figure $2 a, c$ ). The critical value of the Burger number in the quasi-geostrophic case lies in between, $B u_{c}(Q G) \sim 2$. The shallow-water model value, $B u_{c}(R o)$, is given in table 2 for several values of $R o$. For small $R o$, it can be approximated as follows. We know that baroclinic instability occurs when the characteristic scale of motion is larger than the deformation radius (Pedlosky 1987). For a vortex concentrated in the upper layer, we choose a local radius of deformation as that based on the upper layer only, $\dagger$ at the centre of the vortex

$$
L \geqslant R d_{l o c}=\sqrt{g^{\prime} \bar{h}_{1}(r=0)} / f .
$$

The corresponding (theoretical) Burger number for the onset of baroclinic instability is

$$
B u_{t h}=\frac{g^{\prime} H}{f^{2} R d_{l o c}^{2}}=\frac{H}{\bar{h}_{1}(r=0)}=\frac{H}{H_{1}-\bar{\eta}(r=0)} .
$$

We use the cyclogeostrophic balance to obtain an order of magnitude of the interface deviation at the center of the vortex:

$$
\bar{\eta}(r=0) / H \sim(1-\kappa) R o(1+(1+\kappa) R o) / B u_{c}(Q G) .
$$

With $\kappa=0$, we obtain an approximation of $B u_{t h}$ in dimensionless form

$$
B u_{t h} \sim \frac{B u_{c}(Q G)}{\hat{H}_{1} B u_{c}(Q G)-R o-R o^{2}} .
$$

$\dagger$ Note that choosing a two-layer radius of deformation would lead, by an equivalent calculation, to the same physical result, but with far less asymmetry between cyclones and anticyclones. 


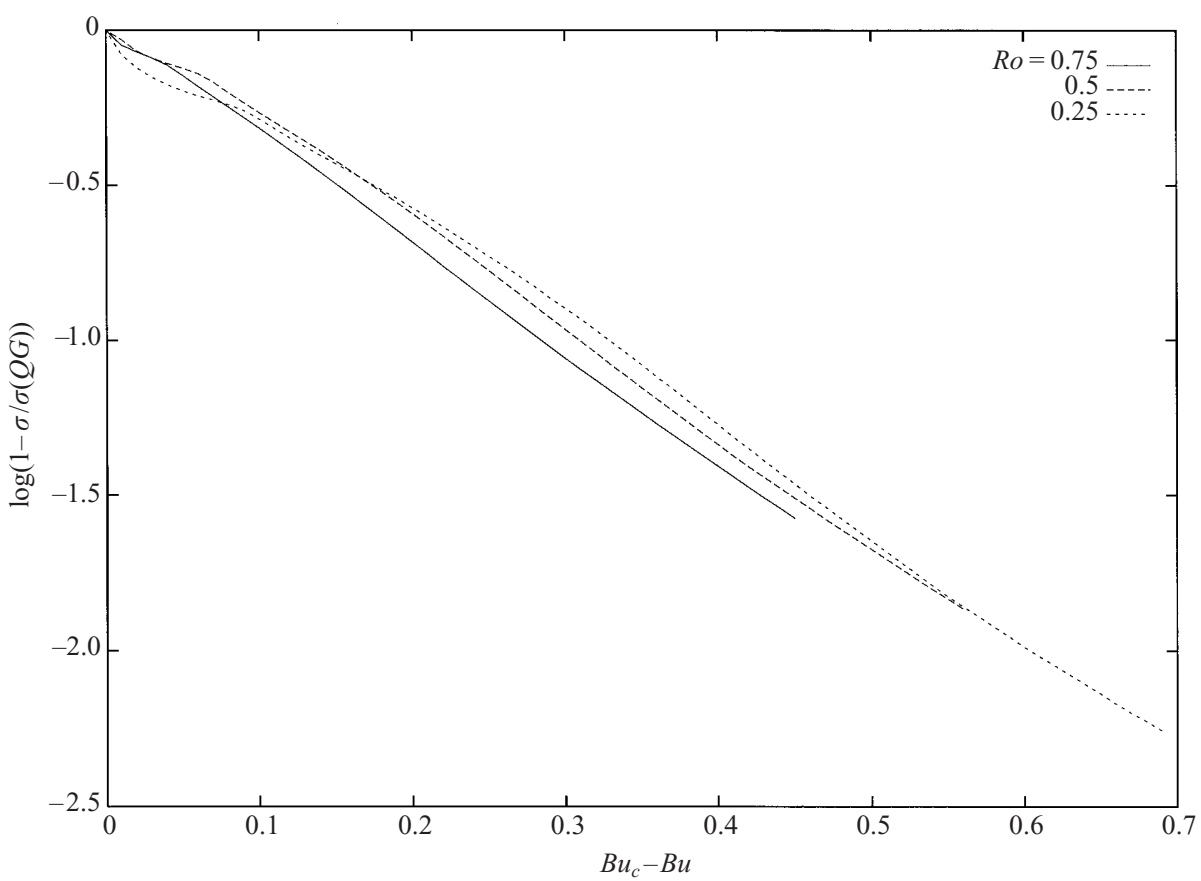

FIGURE 5. Difference between shallow-water and quasi-geostrophic growth rates: $\log (1-\sigma / \sigma(Q G))$ versus $B u_{c}-B u$ for $R o=-0.75,-0.5,-0.25 ; l=2, \alpha=2, \kappa=0, \hat{H}_{1}=0.5$.

The agreement between $B u_{c}$ and $B u_{t h}$ is fair (see table 2). But note that this formula was derived for the present case; it should not be applied directly for other parameters. Physically, the stronger instability of cyclones compared with anticyclones is a general phenomenon. Note finally that for stronger barotropic $(\alpha=3)$ or baroclinic $(\kappa=-1)$ instabilities, several families of unstable eigenmodes exist and overlap in $B u$ space so that no value of $B u_{c}$ can be found.

Following Stegner \& Dritschel (2000), the growth rate of elliptical perturbations could assume a simple variation with respect to $B u$ in the vicinity of $B u_{c}$. The plot of $\log [(\sigma-\sigma(Q G)) / \sigma(Q G)]=f\left(B u_{c}-B u\right)$ for three values of $R o$ (all correspond to anticyclones, for enough values of $\sigma$ were available) is shown on figure 5 . The variation is nearly linear, with slope $\sim-3.3$. Further work could strengthen this result by extending its range of validity.

\subsubsection{Large Burger number limit}

Figure $2(b, d)$ indicates that cyclones are more unstable than anticyclones at finite $B u$ (near $B u=2$ ); other linear calculations extend this result to larger $B u$ for more sheared vortices, more so as $R o$ grows. In that limit, cyclogeostrophic effects could be responsible for this asymmetry. In Appendix D, a simple model is derived from the linearized potential vorticity anomaly equation for $1 / R o<B u<\infty$. This model contains a cyclone/anticyclone asymmetry in the Ro-order terms for the perturbation. Obviously in exact (incompressible) two-dimensional flows, the cyclone-anticyclone asymmetry disappears. Table 3 presents some values of growth rates in this model when $R o$ is varied: cyclones are indeed more unstable than anticyclones. Nonlinear advection terms in the perturbation could explain the cyclone-anticyclone asymmetry 


$\begin{array}{crrrllll}R o & -0.75 & -0.5 & -0.25 & 0 & 0.25 & 0.5 & 0.75 \\ \sigma \times 10^{2} & 0.29 & 0.54 & 0.82 & 1.1 & 1.37 & 1.63 & 1.9\end{array}$

TABLE 3. Growth rates obtained for various values of $R o$ in the simple model for large $B u(\alpha=2, l=2)$.

\begin{tabular}{cllcc}
$\alpha \backslash \kappa$ & \multicolumn{1}{c}{+1} & +0.5 & 0 & -1 \\
2 & $\mathrm{~S}$ & $\mathrm{~S}+\mathrm{F}$ & BCTP & DPBK \\
3 & BTTP & SITP & DPBK & DPBK \\
4 & DPBK & DPBK & DPBK & DPBK
\end{tabular}

TABLE 4. Effect of baroclinicity $\kappa$ on the nonlinear evolutions of power-exponential vortices perturbed on mode $l=2$, for $R o= \pm 0.1, B u=0.25, \hat{H}_{1}=0.5$. Abbreviations: $\mathrm{S}=$ nonlinearly stable, $\mathrm{F}=$ filamentation, BTTP $=$ barotropic tripole, SITP $=$ surface intensified tripole, $\mathrm{BCTP}=$ baroclinic tripole, $\mathrm{DPBK}=$ dipolar breaking. Vortex polarities $(\mathrm{C}=$ cyclone, $\mathrm{AC}=$ anticyclone $)$ are specified only when they correspond to different nonlinear evolutions.

\begin{tabular}{ccccl}
$\alpha \backslash(\kappa, R o)$ & $(0, \pm 0.1)$ & $(0, \pm 0.25)$ & $(0.5, \pm 0.1)$ & \multicolumn{1}{c}{$(0.5, \pm 0.25)$} \\
2 & BCTP & BCTP & S $+\mathrm{F}$ & S + F(AC), SITP(C) \\
3 & DPBK & BCTP & SITP & SITP + F(AC), DPBK(C) \\
4 & DPBK & DPBK & DPBK & DPBK
\end{tabular}

TABLE 5. Effect of Rossby number $R o$ on the nonlinear evolutions of power-exponential vortices perturbed on mode $l=2$, for two values of the baroclinicity $\kappa=0,0.5$ and for $B u=0.25, \hat{H}_{1}=0.5$. Abbreviations are as for table 4.

in this limit. Again, such simple models must be used with caution since many other effects are neglected and only first-order terms are retained.

\section{Nonlinear evolution of elliptically perturbed circular vortices}

The finite-amplitude regimes of the elliptical perturbation, initially superimposed on a power-exponential shielded vortex, are now analysed in the $(R o, B u)$-plane.

4.1. Phenomenology of nonlinear regimes and their distribution in the parameter space The nonlinear numerical model is initialized with the mean vortex and a very weak monochromatic perturbation (to satisfy the linear theory); this perturbation is in the upper layer:

$$
v_{r}^{\prime}=\epsilon r l \exp \left(-r^{\alpha}\right) \sin (l \theta), \quad v_{\theta}^{\prime}=2 \epsilon r\left(1-\frac{1}{2} \alpha r^{\alpha}\right) \exp \left(-r^{\alpha}\right) \cos (l \theta),
$$

and the pressure is computed via the gradient wind balance. Here $l=2, \epsilon=0.001$. The vertical ratio of layerwise intensity is also $\kappa$, but a $\pi / 4$ vertical phase shift is introduced.

Six main types of behaviour are observed, ranging from strong barotropic to strong baroclinic instabilities (see tables 4-7 for their classification with respect to the physical parameters):

(i) Barotropic dipolar breaking (figure $6 a$ ): the perturbation amplifies continuously with time; the core vortex becomes very elliptical due to the shear and strain exerted by the two satellite vortices located at its tips; the core splits into two poles which propagate away with the satellites as dipoles. More generally, barotropic dipolar breaking occurs when the horizontal shear $\alpha$ is large and only for barotropic vortices 


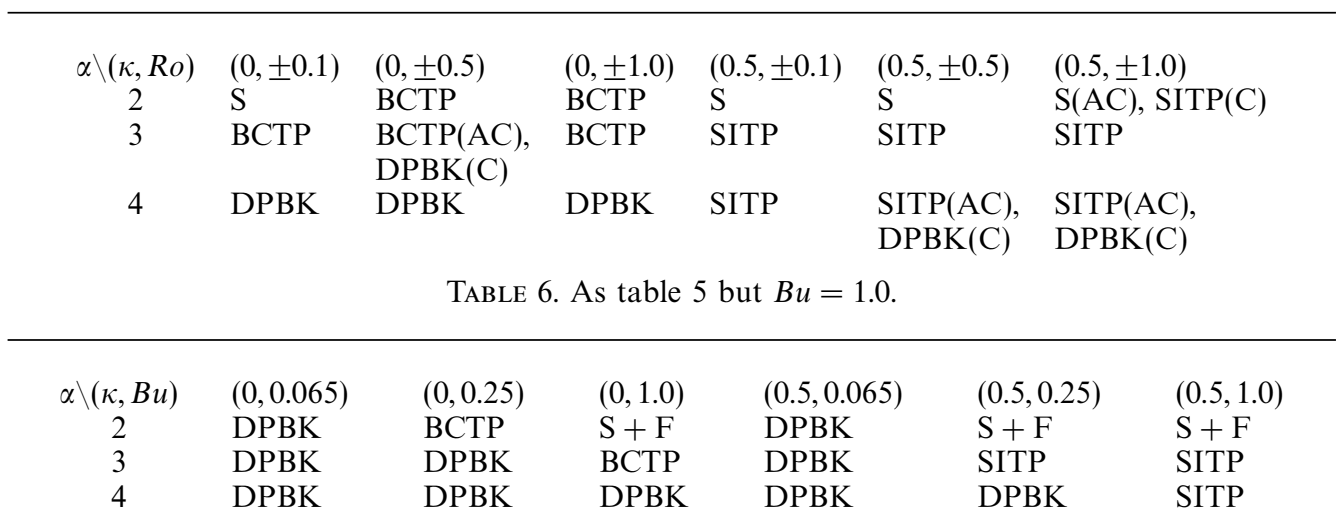

TABLE 7. Effect of Burger number $B u$ on the nonlinear evolutions of power-exponential vortices perturbed on mode $l=2$, for two values of the baroclinicity $\kappa=0,0.5$ and for $R o= \pm 0.1, \hat{H}_{1}=0.5$. Abbreviations are as for table 4.

$(\alpha \geqslant 4, \kappa=1)$. Obviously, this strong barotropic instability does not depend on the Burger number.

(ii) Barotropic tripole $\uparrow$ formation (figure $6 b$ ): the initial evolution is similar to the previous one, but the satellite vortices exert a weaker shear on the core vortex, whose ellipticity remains more moderate. The satellite vortices rapidly reach an equilibrium position on both sides of the core; in the final state, the core-satellite distance is such that the rotation rate of the whole structure and the ellipticity of the core remain constant (cf. Carton \& Legras 1994). More generally, barotropic tripoles form only for moderately unstable barotropic vortices $(\alpha=3, \kappa=1)$. Again, this behaviour does not depend on the Burger number.

(iii) Surface-intensified tripole formation (figure $6 c$ ): in the upper layer, potential vorticity evolves as for the barotropic tripole; in the lower layer, the dynamics are driven by the dominant potential vorticity of the upper layer and by layer coupling via vortex stretching: the lower-layer vortex breaks into three (weak) like-signed poles which align vertically under the upper-layer tripole. More generally, surface-intensified tripoles form when the baroclinic component of the flow is non-zero. They are due to barotropic instability at fairly large $B u$ and $\alpha$. The Rossby number influences this process only for steep cyclones (for which $R o$ must be small to obtain tripoles, e.g. $R o \leqslant 0.1$ when $\alpha=4)$. This can be understood as follows: for cyclones, increasing the Rossby number augments the radial pressure gradient for a given velocity (via cyclogeostrophic balance), more so as the vortex profile is steep. Therefore, for $\kappa \neq 1$, the vertical difference in pressure (or interface displacement) increases with $R o$, which is equivalent to decreasing the Burger number (since $\eta / H \sim R o / B u$ ). Therefore, there must be an upper limit for $R o$ to the formation of surface-intensified tripoles from cyclones.

(iv) Baroclinic tripole formation (figure $6 d$ ): the evolution is similar to that of the surface-intensified tripole, except that the lower-layer vortex breaks into only two satellite vortices; baroclinic instability is stronger than in the previous case, and the evolution becomes closer to baroclinic dipolar breaking. The final structure is

$\dagger$ The term barotropic should not be taken stricto sensu here since the original mean flow is barotropic, but not the perturbation; nevertheless, the final tripole has a negligible baroclinic component 
vertically arch shaped: two pillars (the vertically aligned satellites) are connected in the upper layer by the core vortex. More generally, baroclinic tripoles develop in more baroclinic conditions than the two other types of tripoles (here $\kappa=0$, small $\alpha$ ). To appear, they require opposite-sign potential vorticity anomaly in the two layers. There is also a lower bound on $B u$, below which vortices break into baroclinic dipoles (e.g. $B u=0.25$ for $\alpha=2$ ): baroclinic instability dominates barotropic instability and it is too intense to form a baroclinic tripole. Limits for Ro to form baroclinic tripoles apply to cyclones for $\alpha=3$ (as above).

(v) Baroclinic dipolar breaking (figure 6e): the upper-layer core becomes increasingly elongated in the shear exerted by the satellites in both layers. Finally, the two fragments of the core in the upper layer couple with the lower satellites as two baroclinic dipoles which drift in opposite directions (conserving a null linear momentum). More generally, baroclinic dipoles grow in strongly baroclinic conditions $(\alpha=2$ or $3,-1<\kappa<0.5$, small $B u$ ). For this process, $B u$ needs to be small as $\kappa$ increases. Cyclonic vortices tend to break into baroclinic dipoles more often than anticyclones (confirming the linear stability analysis).

\subsection{Modal analyses and energy budgets}

A modal decomposition of the potential velocity perturbation is performed during the formation and evolution of barotropic, surface-intensified and baroclinic tripoles. For each angular wavenumber $l$, the modal components are defined as

$$
\begin{aligned}
& C_{l}(r, t)=\frac{1}{\pi} \int_{0}^{2 \pi} q_{j}^{\prime}(r, \theta, t) \cos (l \theta) \mathrm{d} \theta, \\
& S_{l}(r, t)=\frac{1}{\pi} \int_{0}^{2 \pi} q_{j}^{\prime}(r, \theta, t) \sin (l \theta) \mathrm{d} \theta,
\end{aligned}
$$

and the modal amplitude is

$$
A_{l}(r, t)=\frac{1}{r_{\max }} \int_{0}^{r_{\max }}\left[C_{l}^{2}(r, t)+S_{l}^{2}(r, t)\right]^{1 / 2} \mathrm{~d} r,
$$

where $q_{j}^{\prime}(r, \theta, t)=q_{j}(r, \theta, t)-q_{j}(r, \theta, t=0)$.

Figure 7 (page 156) shows the modal decomposition for the three kinds of tripoles. For each structure, mode $l=2$ is always dominant in the upper layer. This corresponds to the global tripolar structure and to the elliptical shape of the core vortex. Also note that the period of oscillation of this modal amplitude around the equilibrium value decreases with increasing baroclinicity. A weakly nonlinear theory (e.g. Pedlosky 1987, chap. 7) would be necessary to explain this observation, but it is beyond the scope of the present study. The modes following in intensity are $l=0$ and $l=4$, which directly result from the self-interaction of the initial mode $l=2$. These results are comparable with those of the two-dimensional case (Carton \& Legras 1994), though the formation of the barotropic tripole is less nonlinear than that of the two-dimensional tripole (see also the following paragraph). The more baroclinic the vortex is, the more intense the modification of the mean flow becomes in the lower layer (see previous subsection). In the case of the baroclinic tripole, the finite amplitude of mode $l=4$ indicates that filamentation still occurs at the tips of the ellipse (see figure $6 d$ ) at late times. This filamentation is correlated with a slow alteration of the mean flow. Finally, note that odd modes remain very weak in all the present cases. No asymmetric breaking of the tripoles is observed within the duration of the simulations. 
(a)
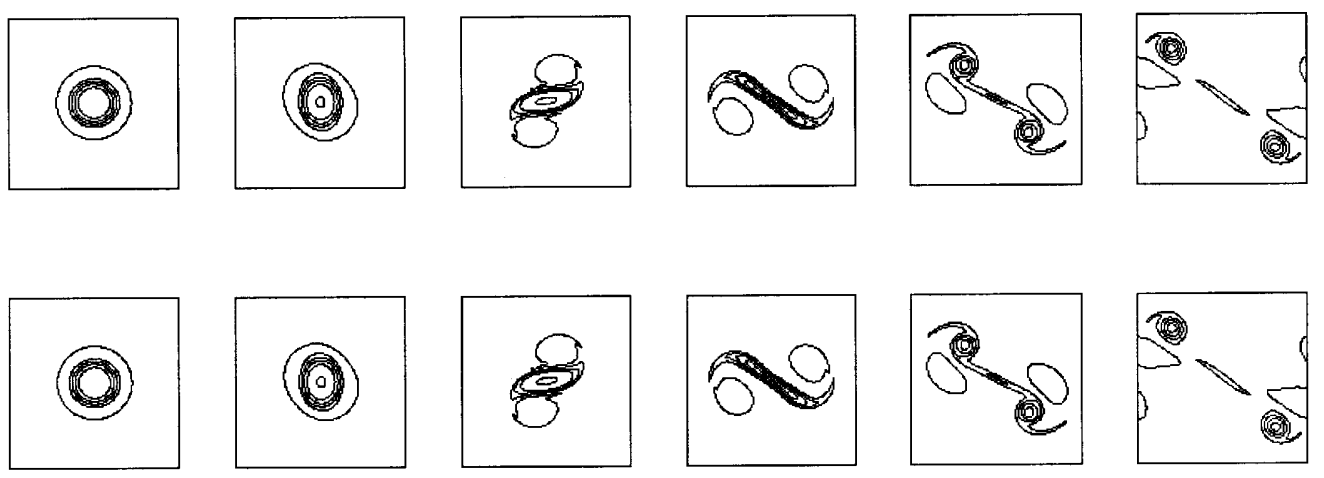

(b)
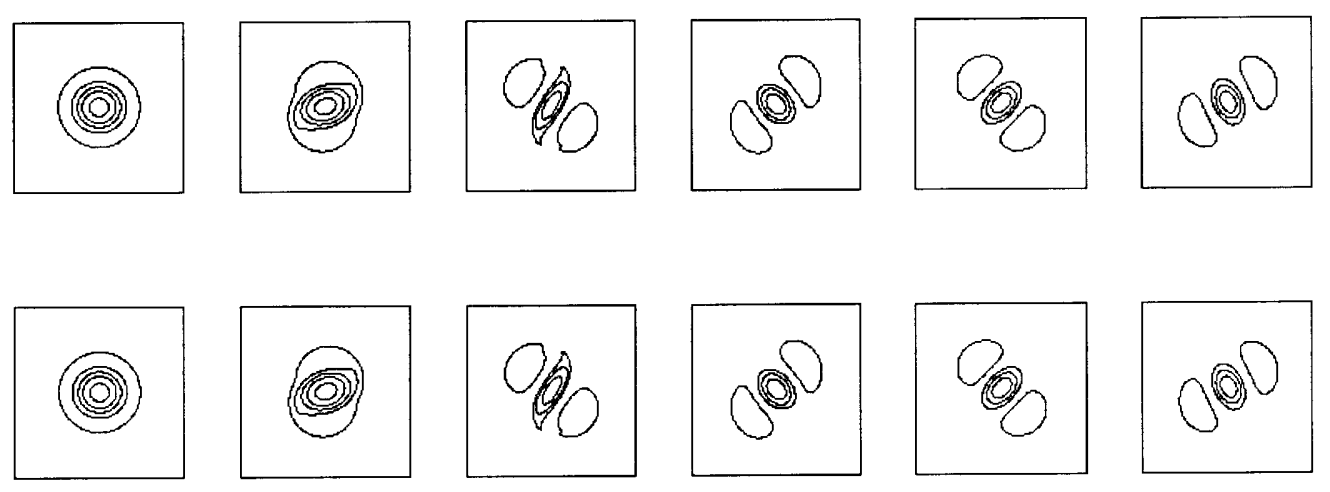

(c)
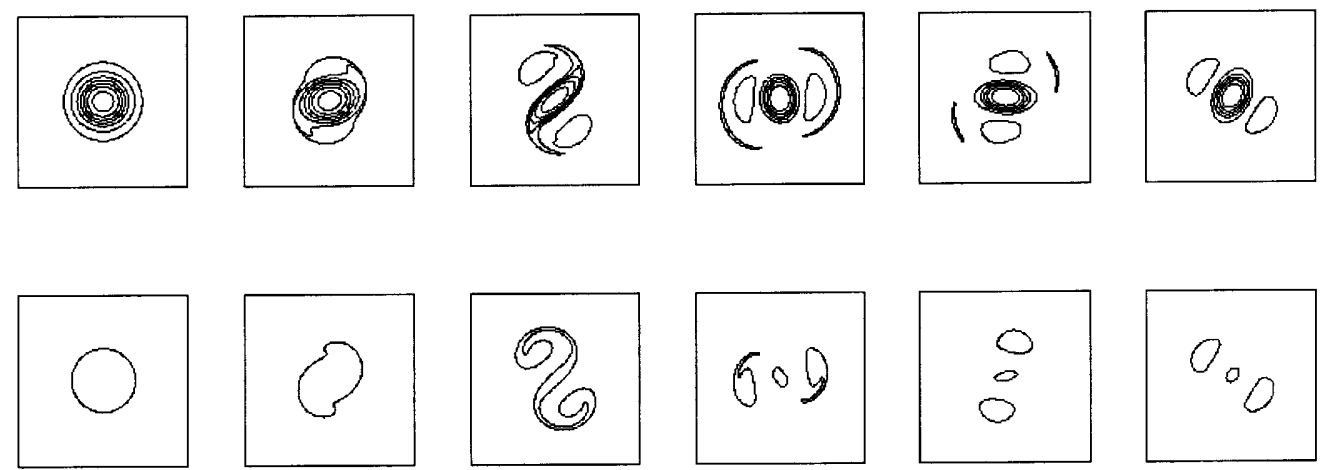

FiguRE $6(a-c)$. For caption see facing page.

The total (kinetic and potential) energy and potential enstrophy were computed for the formation of each type of tripole (not shown). In each case, the total energy in both layers is nearly conserved (within the weak decay due to hyperviscosity). The enstrophy decay is negligible for barotropic tripoles and only weak for the two other types of tripoles. This appears to contrast with the two-dimensional re- 
(d)
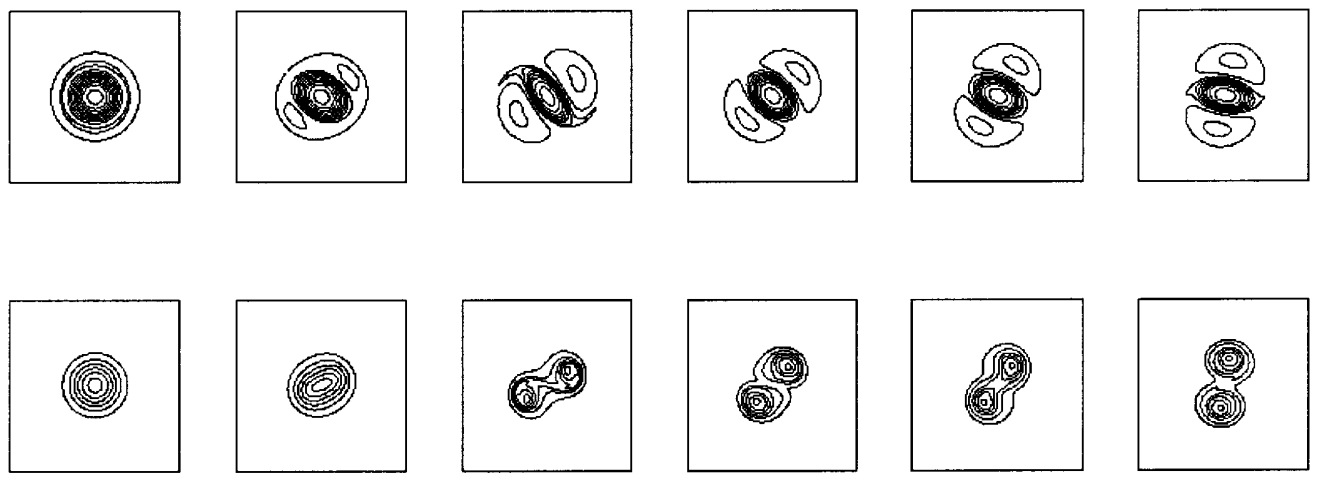

(e)
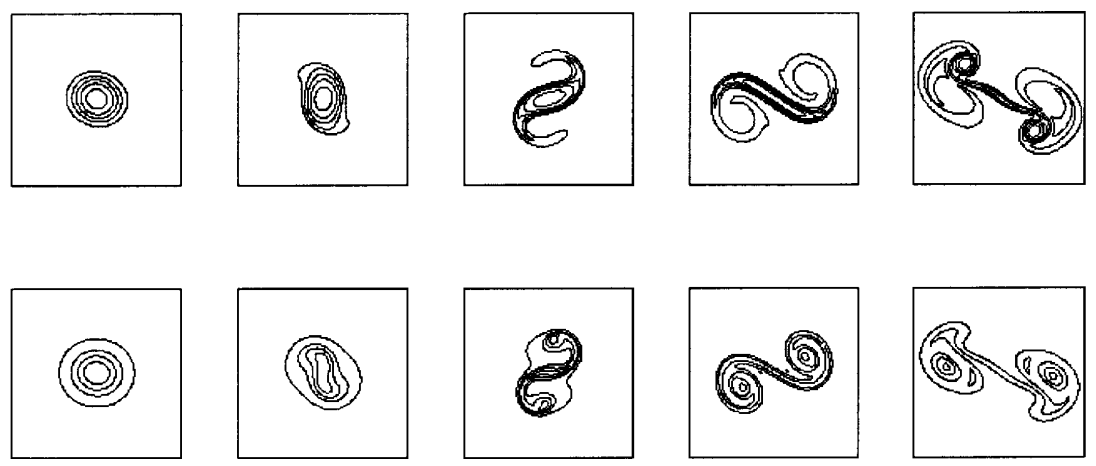

FIGURE 6. Time-evolution of potential vorticity maps showing: $(a)$ the barotropic dipolar breaking of an anticyclonic vortex in the upper and lower layers (upper and lower rows; $R o=0.1, B u=0.25$, $\kappa=1, \alpha=4, l=2$, time interval $=2.4 \tau$, where $\tau$ is the turnover period of the mean vortex at its centre, contour interval $=0.06) ;(b)$ formation of a barotropic tripole $(R o=0.1, B u=0.25, \kappa=1$, $\alpha=3, l=2$, time interval $=2.4 \tau$, contour interval $=0.06) ;(c)$ formation of a surface-intensified tripole $(R o=0.25, B u=0.25, \kappa=0.5, \alpha=3, l=2$, time interval $=12 \tau$, contour interval $=0.15)$; (d) formation of a baroclinic tripole $(R o=0.5, B u=1, \kappa=0, \alpha=3, l=2$, time interval $=8 \tau$, contour interval $=0.15$ for the upper plots and 0.075 for the lower plots); $(e)$ baroclinic dipolar breaking $(R o=0.1, B u=0.25, \kappa=0, \alpha=3, l=2$, time interval $=1.2 \tau$, contour interval $=0.1)$.

sults where a significant enstrophy decrease was observed during tripole formation. This difference is physically related to the weak filamentation occurring here at the tip of the central ellipse. Here the process of tripole formation is less nonlinear than in the two-dimensional case where high modes of deformation reach a substantial amplitude. This may be attributed to the effect of the finite radius of deformation which damps barotropic instability and leads to more linear, oscillatory evolutions for both barotropic and baroclinic instabilities (see the oneand-a-half layer quasi-geostrophic results in Carton et al. 1989, and the two-layer quasi-geostrophic results in Carton \& McWilliams 1996 and Corréard \& Carton 1999).

The energy budgets (see Appendix A) were computed for the formation of the three types of tripoles (see figure $8 a-l$ ). An exponentially growing energy conversion 

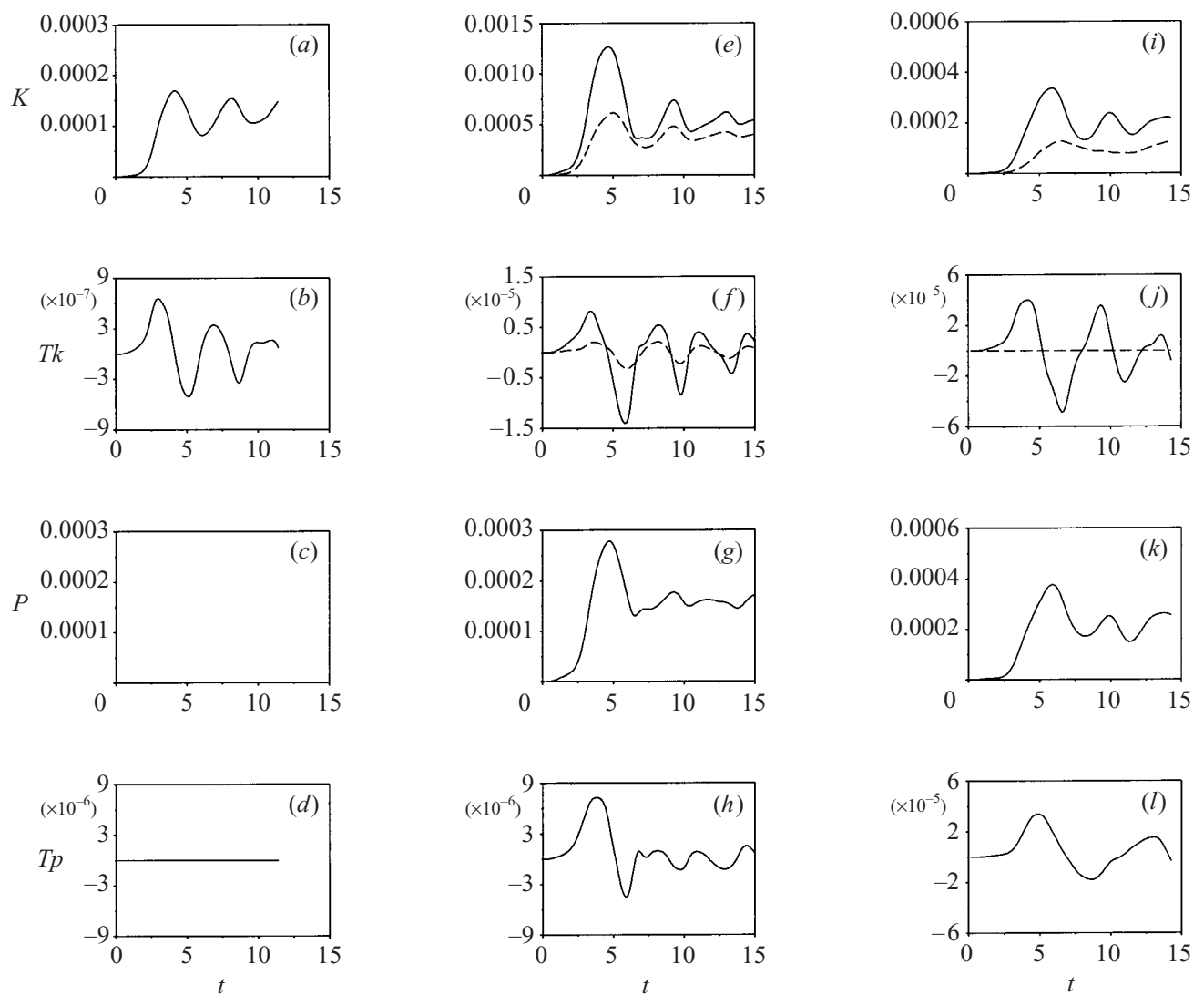

FIGURE 8. Time evolutions of kinetic energy of perturbation $K$, kinetic energy transfer $T k$, potential energy of perturbation $P$ and potential energy transfer $T p$ during the formation of a barotropic tripole $(a-d)$, a surface-intensified tripole $(e-h)$ and a baroclinic tripole $(i-l)$. The time scale is normalized by the rotation period of the initial vortex at its centre. The dashed line indicates the lower layer.

from the mean flow to the perturbation appears at early times as predicted by linear instability theory. The maximum value of this transfer increases with baroclinicity, in agreement with linear theory, and confirming the more intense deformation of baroclinic vortices. During nonlinear stabilization, part of the energy is transferred back from the perturbation to the mean flow. During the subsequent evolution, these transfers change sign quasi-periodically while decreasing in amplitude. The ratio $T p / T k$ increases with baroclinicity, but remains lower than unity, indicating that the instability driving the formation of tripoles is either barotropic or mixed, but never purely baroclinic.

\subsection{Stationarity and stability of tripoles}

If the tripoles resulting from vortex instability are stationary in a uniformly rotating frame of reference, their potential vorticity anomaly should be related to their Bernoulli function. Indeed, replacing the time evolution by the angular variation $\partial_{t}=-\Omega \partial_{\theta}$ (where $\Omega$ is the rotation rate of the tripole), and setting $\hat{v}_{j}=v_{j}-r \Omega$, the 

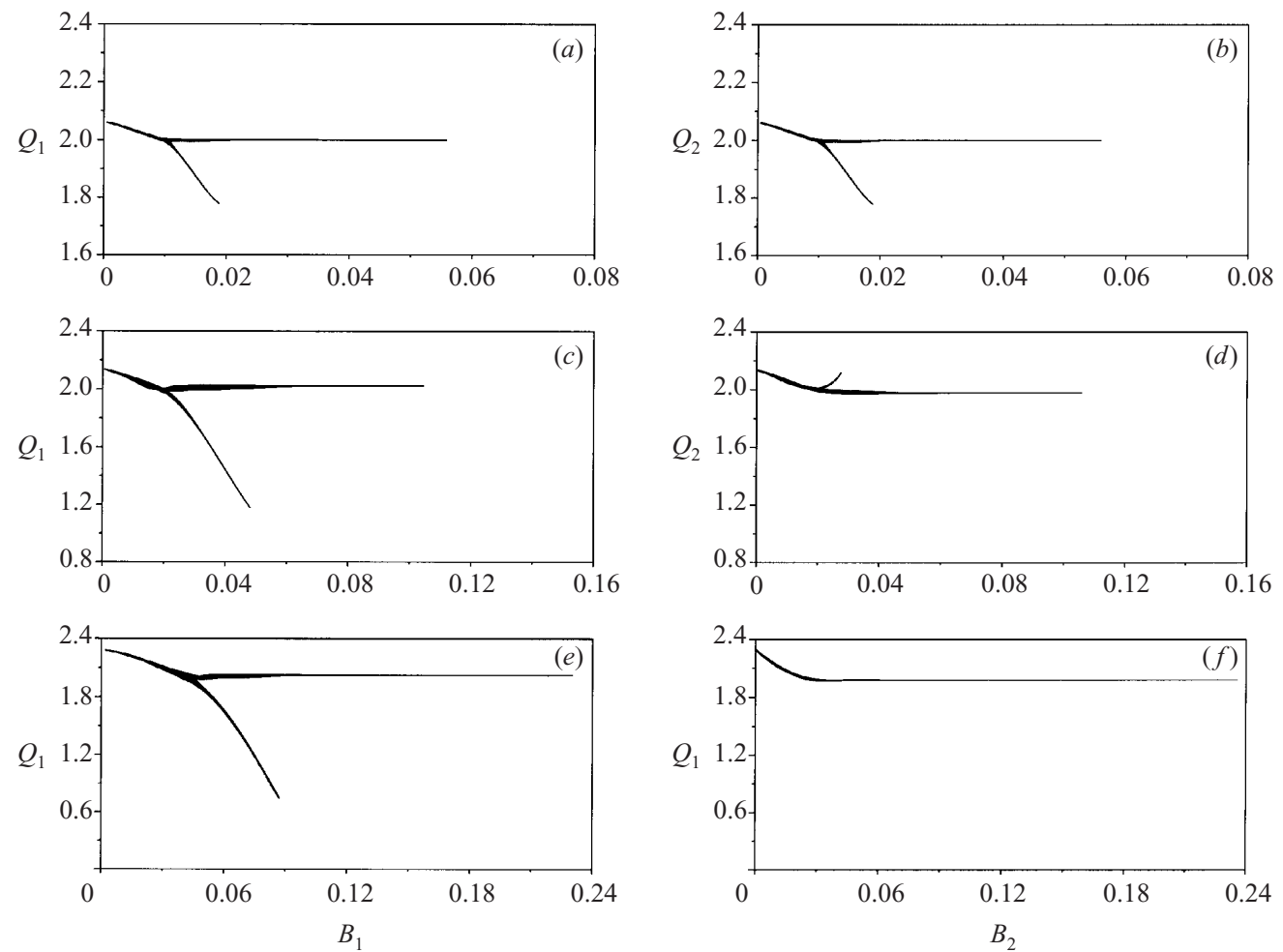

FIGURE 9. Scatter plots of potential vorticity versus Bernoulli function at $t=16 \tau$ for: $(a, b)$ a barotropic tripole $(R o=0.1, B u=0.25, \kappa=1, \alpha=3, l=2)$, with $\Omega=-0.0058 ;(c, d)$ a surface-intensified tripole $(R o=0.25, B u=0.25, \kappa=0.5, \alpha=3, l=2)$, with $\Omega=-0.011$; $(e, f)$ a baroclinic tripole $(R o=0.5, B u=1, \kappa=0, \alpha=3, l=2)$, with $\Omega=-0.0245$. The upper (lower) layer is the left-hand (right-hand) column.

dynamical equations become

$$
\begin{gathered}
\hat{v}_{j}\left(R o \zeta_{j}+1\right)=\partial_{r} B_{j}, \\
u_{j}\left(R o \zeta_{j}+1\right)=-\frac{1}{r} \partial_{\theta} B_{j}, \\
\nabla\left(h_{j} \hat{\boldsymbol{u}}_{j}\right)=0,
\end{gathered}
$$

with

$$
B_{j}=p_{j}+\frac{1}{2} R o\left(u_{j}^{2}+\hat{v}_{j}^{2}\right)-\frac{1}{2} R o \Omega(1+R o \Omega) r^{2},
$$

which is the relative Bernoulli function. These equations are combined to provide the relation $J\left(q_{j}, B_{j}\right)=0$.

The scatter-plot of layerwise potential vorticity anomaly versus Bernoulli function for the barotropic tripole shows three branches, corresponding to the surrounding fluid $\left(q_{j}=0\right)$, to the anticyclonic core of the tripole $\left(q_{j}<0\right)$ and to the cyclonic satellites $\left(q_{j}>0\right.$, see figure $\left.9 a\right)$. In the case of a surface-intensified tripole (figure $\left.9 b\right)$, the upper-layer scatter plot is similar to that of the barotropic tripole. In the lower layer, the two branches with non-zero $q_{j}$ indicate the core and the satellites which are like-signed now. For the baroclinic (arch shaped) tripole, the upper-layer plot is as before, and the single lower-layer branch corresponds to the two identical positive satellites which have aligned vertically under the upper ones. 

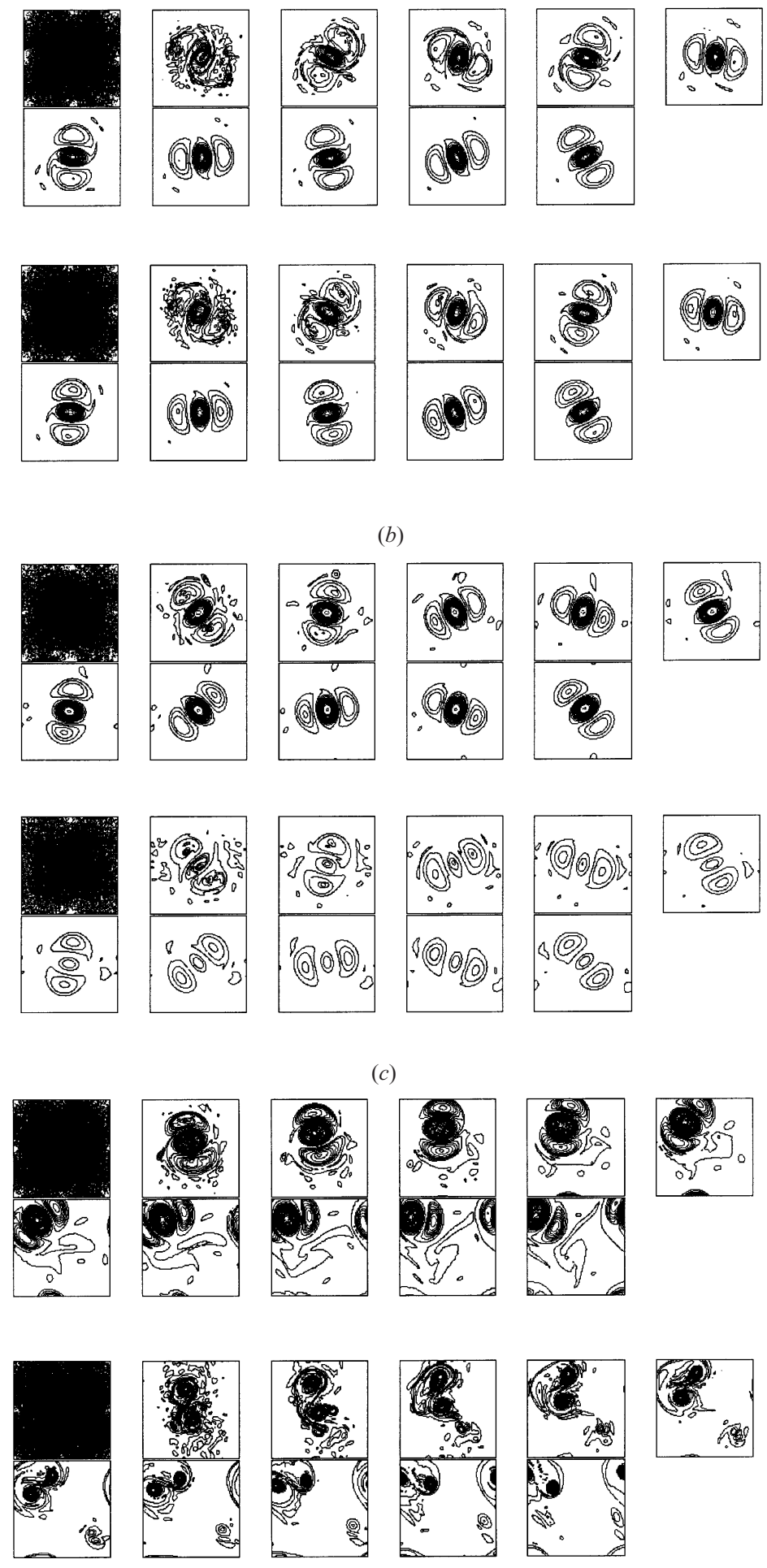

FIGURE 10. For caption see facing page. 
To evaluate the robustness of tripoles, we perturb them with white noise for new model runs. The white noise amplitude in interface deviation is $N L$. For $N L \leqslant 0.025$, barotropic, baroclinic and surface-intensified tripoles rapidly regain their smooth stationary structure. For $N L=0.125$, a mode $l=1$ disturbance grows on the baroclinic tripole which starts drifting; the two other tripoles remain stable (see figure 10). The surface-intensified tripole becomes unstable for $N L=0.25$. The barotropic tripole is the most robust when perturbed by white noise.

\subsection{Ageostrophic and divergent circulations; the Lighthill equation}

Finally, the ageostrophic and divergent circulations are analysed during tripole formation to understand the similarity between quasi-geostrophic and shallow-water model results. The layerwise ageostrophic circulation is, in dimensionless form,

$$
\boldsymbol{v}_{a g}=\boldsymbol{v}-\boldsymbol{k} \wedge \nabla p,
$$

where layer indices have not been added for simplicity. A straightforward analysis of the simulations where tripoles form (see figure 6) shows that this ageostrophic flow is originally circular and finally elliptical and mostly corresponds to the cyclostrophic component of the flow (not shown). Indeed

$$
\boldsymbol{k} \cdot \nabla \wedge \boldsymbol{v}_{a g}=2 \operatorname{RoJ}(u, v) \text {. }
$$

Its amplitude is therefore directly related to the Rossby number.

Since this analysis provides no original information, we compute the horizontal divergence of the velocity:

$$
\nabla_{H} \cdot\left(u_{j}\right)=\nabla^{2} \chi_{j}
$$

using the traditional decomposition

$$
\boldsymbol{u}_{\boldsymbol{j}}=\boldsymbol{k} \wedge \nabla \psi_{j}+\nabla \chi_{j}
$$

Note that this divergence is directly linked to the vertical velocity via the continuity equation:

$$
w_{j}=\frac{\mathrm{d} h_{j}}{\mathrm{~d} t}=-h_{j} \nabla^{2} \chi_{j}
$$

In the quasi-geostrophic model, the divergent circulation is always weak. In the shallow-water model, its evolution is now computed for stable tripoles.

During the formation of a baroclinic tripole, the velocity divergence concentrates in the periphery of the anticyclonic core vortex (figure 11) where the horizontal shear is maximum. The amplitude of the divergence oscillates with time (not shown on the figure which has a large time sampling). These characteristics suggest the presence of an inertial wave (known to be favoured by anticyclonic vorticity). A quadrupolar pattern is rapidly established, showing the alternance of upward and downward flow around the central ellipse. For the barotropic tripole, this concentration in anticyclonic regions and the oscillating amplitude are also observed, but the spatial pattern of velocity divergence is more intricate.

FIGURE 10. Time-evolution of potential vorticity maps showing the effect of a white noise of level NL $=5$ on $(a)$ a barotropic tripole $(R o=0.1, B u=0.25, \kappa=1, \alpha=3$, time interval $=0.64 \tau$, contour interval $=0.06) ;(b)$ a surface-intensified tripole $(R o=0.25, B u=0.25, \kappa=0.5, \alpha=3$, time interval $=0.8 \tau$, contour interval $=0.15) ;(c)$ a baroclinic tripole $(R o=0.5, B u=1, \kappa=0$, $\alpha=3$, time interval $=0.64 \tau$, contour interval $=0.15$ for the upper plots and 0.075 for the lower plots). For each tripole, the two upper (lower) rows correspond to the upper (lower) layer; for each row, the lower panel is the time continuation of the upper one. 

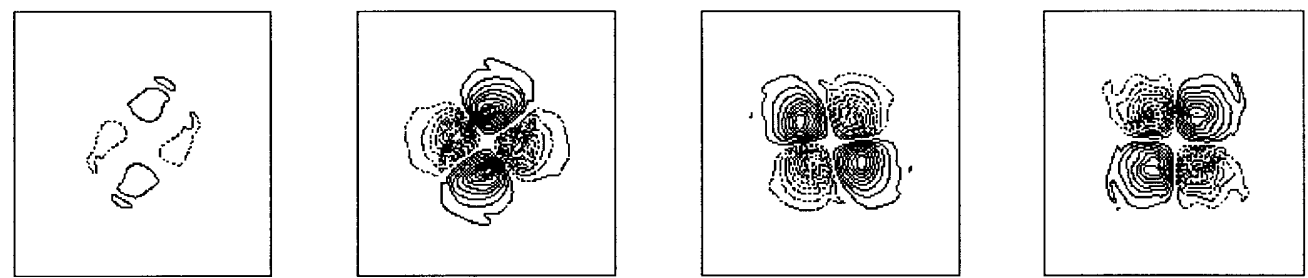

FIGURE 11. Time evolution of the horizontal velocity divergence during the formation of a baroclinic tripole (see Figure $6 d$ ). The first frame is at $t=3 \tau$ and the time interval is $\delta t=6 \tau$. The contour interval is $5 \times 10^{-4}$.

The quadrupolar pattern of velocity divergence for a baroclinic tripole can be explained by using the relative vorticity equation on the $f$-plane:

$$
\frac{\mathrm{d} \zeta_{j}}{\mathrm{~d} t}=\left(1+R o \zeta_{j}\right) \nabla^{2} \chi_{j}
$$

This equation can be simplified, assuming that the flow is stationary, that the velocity is essentially rotational and that the Rossby number is moderate:

$$
J\left(\psi_{j}-\frac{1}{2} \Omega r^{2}, \nabla^{2} \psi_{j}\right)=\nabla^{2} \chi_{j},
$$

where $\Omega$ is the rotation rate of the tripole. Further simplication is based on the circular form of the mean flow and on the weak amplitude of the perturbation:

$$
J\left(\bar{\psi}_{j}-\frac{1}{2} \Omega r^{2}, \nabla^{2} \psi_{j}^{\prime}\right)+J\left(\psi_{j}^{\prime}, \nabla^{2} \bar{\psi}_{j}\right)=\nabla^{2} \chi_{j},
$$

The Jacobian of the perturbation streamfunction with its relative vorticity is one order smaller than the two terms retained on the left-hand side of this equation, and is thus neglected. This final equation demonstrates that the vertical velocity has the same angular structure as the dominant rotational mode of perturbation, here elliptical in the upper layer (note that the nonlinear correction to the mean flow has a null contribution to the velocity divergence in this equation).

Quantitatively, the two terms of (6) have been computed numerically and subtracted. This difference also has an elliptical shape and amounts to $1.6 \%$ of the velocity divergence. This justifies the approximations leading to (6).

In all cases, this velocity divergence is very weak compared with relative vorticity (the rotational part of the flow); the ratio $\left|\nabla^{2} \chi / \nabla^{2} \psi\right|$ is at most $10^{-3}$. This also explains why little difference is observed between quasi-geostrophic and shallow-water tripole formation and evolution.

Finally, information on the propagating part of the divergent circulation can be gained from the Lighthill equation (Lighthill 1952). It is derived from the momentum and continuity equations (1) and (2) and is written in dimensionless form:

$$
\begin{aligned}
2 R o^{2} \partial_{t}^{3} \eta & +2 \partial_{t} \eta-\frac{B u}{2} \partial_{t} \nabla^{2} \eta=R o B u \partial_{t}\left(D S_{2}-D S_{1}\right) \\
& -R o \partial_{t} \nabla \cdot\left[\eta \nabla\left(p_{1}+p_{2}\right)\right]+\frac{B u}{2}\left[\boldsymbol{u}_{2} \cdot \nabla \zeta_{2}-\boldsymbol{u}_{1} \cdot \nabla \zeta_{1}\right] \\
& +\frac{B u}{2}\left[D_{2} \zeta_{2}-D_{1} \zeta_{1}\right]-\nabla \cdot\left[\eta\left(\boldsymbol{u}_{2}-\boldsymbol{u}_{1}\right)\right] \\
& +\operatorname{Ro}_{t}\left[\partial_{x}\left[\eta\left(v_{1}+v_{2}\right)\right]-\partial_{y}\left[\eta\left(u_{1}+u_{2}\right)\right],\right.
\end{aligned}
$$

where $D_{j}=\partial_{x} u_{j}+\partial_{y} v_{j}, D S_{j}=\partial_{x}^{2}\left(h_{j} u_{j}^{2}\right)+2 \partial_{x y}^{2}\left(h_{j} u_{j} v_{j}\right)+\partial_{y}^{2}\left(h_{j} v_{j}^{2}\right)$. This equation is applied 
(a)
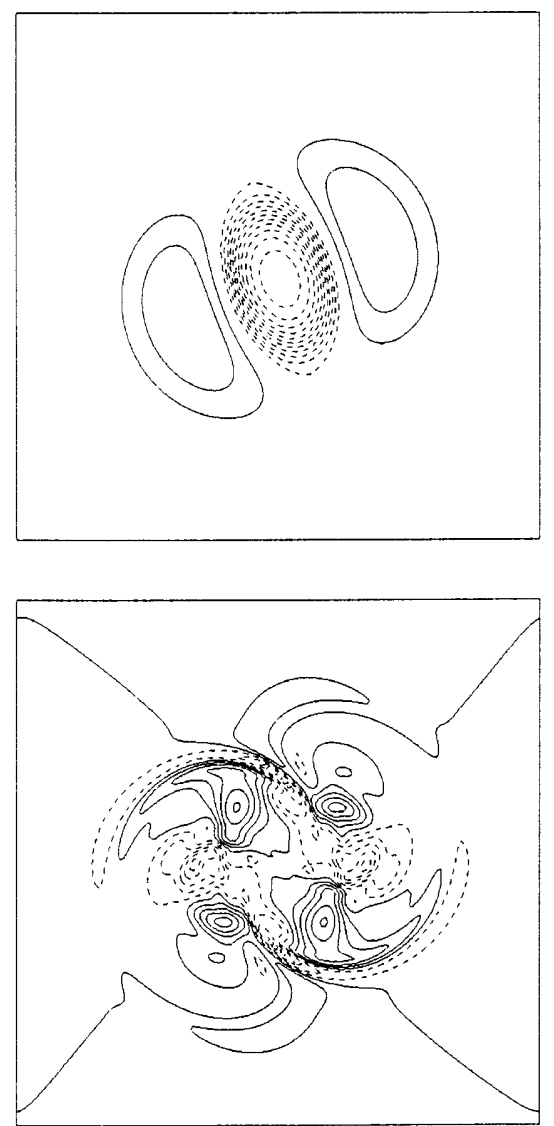

(b)
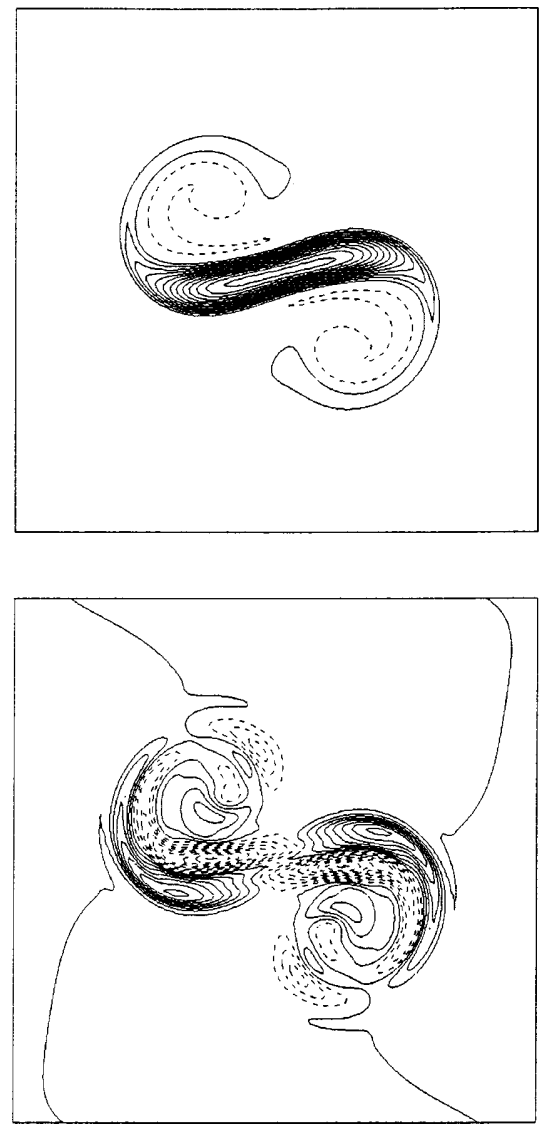

FIGURE 12. Comparison of upper-layer potential vorticity and the Lighthill term for the generation of internal gravity waves, $(a)$ for the baroclinic tripole of figure $6(d)$ at time $t=19 \tau$, and $(b)$ for the formation of a baroclinic dipole of figure $6(e)$ at time $t=3.8 \tau$. Contour interval is 0.1 for potential vorticity (upper plots) and 0.001 for the Lighthill term (lower plots).

to the formation of a baroclinic tripole (example of figure $6 d$ ) and to baroclinic dipolar breaking in the frontal limit (example of figure 6e). In both cases, the Lighthill term for the generation of internal gravity waves (the right-hand side of (7)) is the weak (on order of $5 \times 10^{-3}$ times the upper-layer potential vorticity maximum) and it is quadrupolar (see figure 12 and also Polvani et al. 1994). Thus, little generation of gravity waves occurs even during energetic events such as dipolar breaking. This confirms our previous analysis of a weak divergent flow.

\section{More complex multipoles? Conclusions}

A similar stability study has been conducted for the same family of isolated vortices perturbed with a triangular wave (mode $l=3$ ). Linear instability characteristics of this mode are very similar to those of the elliptical mode as stated in $\S 3$. Triangular perturbations can also stabilize nonlinearly at finite amplitude and form barotropic, or surface-intensified, or baroclinic quadrupoles, with simple $(q, B)$ relations; they are more short-lived than comparable tripoles. Their decay occurs asymmetrically, due 
to the interaction of elliptical and triangular waves which have similar growth rates. Numerically, more complex multipoles than quadrupoles were not observed as robust states.

In summary, the study of isolated vortex stability in a two-layer rotating shallowwater fluid has shown a striking similarity with quasi-geostrophic model results, a similarity already noticed by Stegner \& Dritchel (2000). Asymmetry between cyclones and anticyclones was found mostly in the frontal limit, and to a lesser degree, for large Burger numbers. In both cases, physical arguments (difference of radial gradients of mean PHA in the frontal case, and/or cyclostrophic asymmetry of perturbation velocity) and simple models partially accounted for the slightly stronger instability of cyclones. This weak asymmetry may seem surprising considering that strong parity bias was often found in intermediate models (Tang 1989; Sutyrin 1994; Stegner \& Zeitlin 1995); however, exaggerated parity bias in intermediate models compared with the shallow-water model has been recently attributed to the truncated dynamics of these intermediate models (Ben Jelloul \& Carton 2001). A stronger instability of cyclones than of anticyclones was also found for a divergent elliptic vortex in one-and-a-half layer shallow-water flow (Arai \& Yamagata 1994).

The nonlinear evolution of these isolated vortices, elliptically perturbed, confirmed this similarity between quasi-geostrophic and shallow-water evolution. It also showed that formation of tripoles with various vertical structures was not an exceptional occurrence. Note that tripoles can also form from piecewise-constant vortices, elliptically perturbed, in a two-layer rotating shallow-water model (Baey \& Carton 2001). Shallow-water tripoles are stationary in rotation and long lived like their quasi-geostrophic counterparts (Correard \& Carton 1999). The weakness of the ageostrophic and divergent circulations during shallow-water tripole formation and evolution may account for the similarity between the two models.

Tripole formation was also shown not to substantially dissipate energy nor enstrophy. It could be of interest to investigate if tripole formation and dipolar breaking correspond to noticeably different angular momentum distributions in the initial circular vortices leading to these final states (see Carton \& Legras 1994).

A complex relation was found between the rotating tripole Bernoulli function and potential vorticity anomaly. From this relation, the mathematical form of the spatial vorticity distribution of the tripole could be derived (though this appears very difficult). With such an expression, the tripole parameters (amplitude and radius) could be related to those of the original vortex and a rule could be established on the possibility of a transition between these two states. Again, this prospective task seems particularly arduous.

In conclusion, vortex stability, at least in our parameter ranges, is not a good candidate to explain the parity bias in oceanic vortices. For instance, though Mediterranean water eddies have sometimes been observed to form as dipoles over the Portimao canyon south of Portugal (Serra \& Ambar 2001), their cyclonic counterpart has rarely been found beyond a few hundred miles away from the formation sites (Carton et al. 2002). Vortex interaction with waves, topography, beta-effect and large-scale currents should thus be investigated to explain this asymmetry in the ocean.

This work was performed at SHOM/CMO and at IFREMER/LPO; it is a contribution to both institutes' research program "Mesoscale turbulent processes in the Northeastern Atlantic Ocean". The first author worked under SHOM/CMO contract 787007-004702925. The authors thank Professors W. K. Dewar, J. C. McWilliams, D. G. Dritschel and G. R. Flierl, and Drs L. Hua and P. Klein for fruitful discussions, 
and three anonymous referees whose suggestions helped improve the manuscript. We dedicate this work to the memory of our friend and colleague, Dr Rupert Ford.

\section{Appendix A. Energy transfers}

Multiplying the nonlinear momentum and continuity equations for the perturbation by the complex conjugates of $\boldsymbol{u}_{j}^{\prime}, \eta^{\prime}=p_{2}^{\prime}-p_{1}^{\prime}$ we obtain

$$
\begin{aligned}
\int_{0}^{\infty} & r \mathrm{~d} r\left[\sum_{j=1}^{2} \bar{h}_{j} \frac{\mathrm{d}}{\mathrm{d} t}\left\langle K_{j}^{\prime}\right\rangle+\frac{\mathrm{d}}{\mathrm{d} t}\left\langle P^{\prime}\right\rangle\right] \\
= & \sum_{j=1}^{2} T K_{j}+T P+\int_{0}^{\infty}\left\langle\frac{\eta^{\prime 2}}{B u}\left[\partial_{r} u_{1}^{\prime}+\frac{u_{1}^{\prime}}{r}+\frac{1}{r} \partial_{\theta} v_{1}^{\prime}\right]\right\rangle r \mathrm{~d} r \\
& -\int_{0}^{\infty}\left\langle p_{2}^{\prime}\left[\left(\sum_{j=1}^{2} \partial_{r}\left(r u_{j}^{\prime} h_{j}^{\prime}\right)\right)-\partial_{\theta}\left(\eta^{\prime} v_{1}^{\prime}\right)\right]\right\rangle \mathrm{d} r
\end{aligned}
$$

with $K_{j}^{\prime}=\left|u_{j}^{\prime}\right|^{2}+\left|v_{j}^{\prime}\right|^{2}, P^{\prime}=\left|\eta^{\prime}\right|^{2} / B u, h_{j}^{\prime}=(-1)^{j} \eta^{\prime}$ and the angular average

$$
\langle a\rangle=\int_{0}^{2 . \pi} a \mathrm{~d} \theta
$$

The kinetic and potential rates of energy conversions and the baroclinic conversion are

$$
\begin{gathered}
T K_{j}=-\int_{0}^{\infty}\left(\frac{\mathrm{d} \bar{v}_{j}}{\mathrm{~d} r}-\frac{\bar{v}_{j}}{r}\right)\left\langle u_{j}^{\prime} v_{j}^{\prime}\right\rangle \bar{h}_{j} r \mathrm{~d} r, \quad j=1,2, \\
T P=\int_{0}^{\infty}\left(\bar{v}_{1}-\bar{v}_{2}\right)\left\langle p_{2}^{\prime} \partial_{\theta} \eta^{\prime}\right\rangle \mathrm{d} r, \\
T P K=\sum_{j=1}^{2} \int_{0}^{\infty}\left\langle p_{j}^{\prime}\left(\partial_{r}\left(r \bar{h}_{j} u_{j}^{\prime}\right)+\bar{h}_{j} \partial_{\theta}\left(v_{j}^{\prime}\right)\right)\right\rangle \mathrm{d} r ;
\end{gathered}
$$

the last two terms on the right-hand side of the energy equation are a nonlinear contribution of the perturbation.

\section{Appendix B. Linear instability in the frontal limit}

In the frontal limit $(R o / B u \sim 1)$, the shallow-water conservation of potential height anomaly

$$
\frac{\mathrm{d} \bar{\theta}_{j}}{\mathrm{~d} r}=0, \quad \bar{\theta}_{j}=\frac{\bar{\eta}_{j} / B u-\bar{\zeta}_{j}}{1+R o \bar{\zeta}_{j}}
$$

is linearized, assuming geostrophy for the normal-mode perturbation; this implies that $R o$ is small, which is compatible with $R o / B u \sim 1$ when $B u$ is also small. The resulting equation is

$$
\left(\bar{v}_{j}-r c\right) \theta_{j}^{\prime}-\frac{\mathrm{d} \bar{\theta}_{j}}{\mathrm{~d} r} p_{j}^{\prime}=0, \quad \theta_{j}^{\prime}=\frac{\eta_{j}^{\prime} / B u-\bar{\theta}_{j} \zeta_{j}^{\prime}}{1+R o \bar{\zeta}_{j}},
$$

with $\zeta_{j}^{\prime}=\nabla^{2} p_{j}^{\prime}, \eta_{j}^{\prime}=(-1)^{j} \eta^{\prime} / \hat{H}_{j}$. 
This equation is solved by a matrix method with the condition of null perturbation at the origin and at infinity.

\section{Appendix C. Shallow-water perturbation velocity in the linear theory}

Using the linearized (layerwise) shallow-water equations, and assuming that the pressure is normalized by the layer density, the expression for radial and azimuthal perturbation velocities is, in dimensionless form,

$$
\begin{aligned}
& u_{j}^{\prime}=\frac{\mathrm{il}\left[\operatorname{Ro}\left(c-\bar{v}_{j} / r\right) \mathrm{d} p_{j}^{\prime} / \mathrm{d} r-\left(1+2 R o \bar{v}_{j} / r\right) p_{j}^{\prime} / r\right]}{\left(1+2 R o \bar{v}_{j} / r\right)\left(1+R o \bar{v}_{j} / r+R o \mathrm{~d} \bar{v}_{j} / \mathrm{d} r\right)-l^{2} R o^{2}\left(c-\bar{v}_{j} / r\right)^{2}}, \\
& v_{j}^{\prime}=\frac{\left(1+R o \bar{v}_{j} / r+R o \mathrm{~d} \bar{v}_{j} / \mathrm{d} r\right) \mathrm{d} p_{j}^{\prime} / \mathrm{d} r-l^{2} R o^{2}\left(c-\bar{v}_{j} / r\right) p_{j}^{\prime} / r}{\left(1+2 R o \bar{v}_{j} / r\right)\left(1+R o \bar{v}_{j} / r+R o \mathrm{~d} \bar{v}_{j} / \mathrm{d} r\right)-l^{2} R o^{2}\left(c-\bar{v}_{j} / r\right)^{2}} .
\end{aligned}
$$

Setting $R o=0$, we immediately obtain the quasi-geostrophic form.

\section{Appendix D. Linear instability in the large- $B u$ limit}

For $1 / R o<B u<\infty$, we neglect the $1 / B u$ terms in front of the $R o$-order correction terms. Then, the layers decouple and a quasi-two-dimensional equation is obtained for the normal mode perturbation:

$$
(\bar{v}-r c) \zeta^{\prime}-\frac{\mathrm{d} \bar{\zeta}}{\mathrm{d} r} p^{\prime}=0 .
$$

Due to our choice of mean flow, $\bar{\zeta}$ is antisymmetric for cyclones and anticyclones. But due to the (linearized) gradient wind approximation, the perturbation vorticity is not antisymmetric:

$$
\zeta^{\prime}=\nabla^{2} p^{\prime}-2 \operatorname{Ro} J\left(u^{\prime}, \bar{v}\right) \text {. }
$$

This equation is solved by a matrix method with condition of null perturbation at the origin and at infinity.

\section{REFERENCES}

Arai, M. \& Yamagata, T. 1994 Asymmetric evolution of eddies in rotating shallow water. Chaos 4, $163-176$

Baey, J. M. \& CARTON, X. J. 2001 Piecewise-constant vortices in two-layer rotating shallow-water flows. Proc. IUTAM Symp. on Advances in Mathematical Modeling of Atmosphere and Oceans, pp. 87-92. Kluwer.

Ben Jelloul, M. \& Carton, X. J. 2001 Asymptotic models and application to vortex dynamics. Proc. IUTAM Symp. on Advances in Mathematical Modeling of Atmosphere and Oceans, pp. 105-110. Kluwer.

Baraille, R. \& Filatoff, N. G. 1995 Le modèle shallow-water multicouches isopycnal de Miami. EPSHOM/CMO Res. Rep. 03/95. Brest, France.

Carnevale, G. F. \& Kloosterziel, R. C. 1994 Emergence and evolution of triangular vortices. J. Fluid Mech. 259, 305-331.

Carton, X. J., Chérubin, L. M., Paillet, J., Morel, Y., Serpette, A. \& Le Cann, B. 2002 Meddy coupling with a deep cyclone in the Gulf of Cadiz. To appear in J. Mar. Syst.

Carton, X. J., Flierl, G. R. \& Polvani, L. M. 1989 The generation of tripoles from unstable axisymmetric vortex structures. Europhys. Lett. 9, 339-344.

Carton, X. J. \& Legras, B. 1994 The life-cycle of tripoles in two-dimensional incompressible flows. J. Fluid Mech. 267, 53-82. 
Carton, X. J. \& MCWilliams, J. C. 1996 Nonlinear oscillatory evolution of a baroclinically unstable geostrophic vortex. Dyn. Atmos. Oceans 24, 207-214.

CORRÉARD, S. M. \& CARTON, X. J. 1999 Formation and stability of tripolar vortices in stratified geostrophic flows. Il Nuovo Cimento C 22, 767-777.

Dewar, W. K., Blundell, J. R. \& Killworth, P. D. 1999 Primitive equation instability of wide oceanic rings. Part II: Numerical studies of ring stability. J. Phys. Oceanogr. 29, 1744-1758.

Dewar, W. K. \& Killworth, P. D. 1995 On the stability of oceanic rings. J. Phys. Oceanogr. 25, $1467-1487$.

FLIERL, G. R. 1978 Models of vertical structure and the calibration of two-layer models. Dyn. Atmos. Oceans 2, 341-381.

FORD, R. 1994 The instability of an axisymmetric vortex with monotonic potential vorticity in rotating shallow water. J. Fluid Mech. 280, 303-334.

van Heijst, G. J. F., Kloosterziel, R. C. \& Williams, C. W. M. 1991 Laboratory experiments on the tripolar vortex in a rotating fluid. J. Fluid Mech. 225, 301-331.

Holton, J. R. 1992 An Introduction to Dynamic Meteorology. Academic.

Killworth, P. D., Blundell, J. R. \& Dewar, W. K. 1997 Primitive equation instability of wide oceanic rings. Part I: Linear theory. J. Phys. Oceanogr. 27, 941-962.

Lighthill, M. J. 1952 On sound generated aerodynamically. I. General theory. Proc. R. Soc. Lond. A 211, 564-587.

Morel, Y. G. \& CARTON, X. J. 1994 Multipolar vortices in two-dimensional incompressible flows. J. Fluid Mech. 267, 23-51.

Orlandi, P. \& van HeiJst, G. J. F. 1992 Numerical simulations of tripolar vortices in 2D flows. Fluid Dyn. Res. 9, 179-206.

Pedlosky, J. 1987 Geophysical Fluid Dynamics. Springer.

Polvani, L. M., Mc Williams, J. C., Spall, M. A. \& Ford, R. 1994 The coherent structures of shallow-water turbulence: deformation-radius effects, cyclone/anticyclone asymmetry and gravity-wave generation. Chaos 4, 177-186.

Serra, N. \& Ambar, I. 2001 Eddy generation in the Mediterranean undercurrent. To appear in Deep-Sea Res.

Sokolovskiy, M. A. \& Verron, J. 2000 Finite-core hetons; stability and interactions. J. Fluid Mech. 423, $127-154$.

Stegner, A. \& Dritschel, D. G. 2000 A numerical investigation of the stability of isolated shallow-water vortices. J. Phys. Oceanogr. 30, 2562-2573.

Stegner, A. \& Zeitlin, V. 1995 What can asymptotic expansions tell us about large-scale quasigeostrophic anticyclonic vortices? Nonlinear Proc. Geophys. 2, 186-193.

SuTYRIN, G. G. 1994 Long-lived planetary vortices and their evolution: conservative intermediate geostrophic models. Chaos 4, 203-212.

TANG, B. Y. 1989 Numerical modeling of geostrophic turbulence and eddy evolution using generalized geostrophic equations. PhD thesis, The Florida State University, Tallahassee, USA. 